\title{
DYNAMICAL DISORDER AND SELF-CORRELATION IN THE CHARACTERIZATION OF NONLINEAR SYSTEMS. APPLICATION TO DETERMINISTIC CHAOS
}

\author{
JUAN ANTONIO HERNÁNDEZ*, ROSA MARÍA BENITO' \\ and JUAN CARLOS LOSADA ${ }^{\ddagger}$ \\ Grupo de Sistemas Complejos. ETSI Agrónomos, \\ Universidad Politécnica de Madrid, \\ Ciudad Universitaria s/ $n$, Madrid, 28040, Spain \\ *jantonio hernandezibiberbanda.es \\ trosamaria benito@upm.es \\ †uancarlos.losada@upm.es
}

\begin{abstract}
A new methodology to characterize nonlinear systems is described. It is based on the measurement over the time series of two quantities: the "Dynamical order" and the "Self-correlation". The averaged "Scalar" and "Perpendicular" products are introduced to measure these quantities. While this approach can be applied to general nonlinear systems, the aim of this work is to focus on the characterization and modeling of chaotic systems. In order to illustrate the method, applications to a two-dimensional chaotic system and the modeling of real telephony traffic series are presented. Three important aspects are discussed: the use of the averaged "Scalar" product as supplement of the "Lyapunov exponent", the use of the averaged "Perpendicular" product as a refinement of the "Mutual information" and the reduction of $m$-dimensional systems to the study of only one dimension. This new conceptual framework introduces a perspective to characterize real and theoretical processes with a unifying method, irrespective of the system classification.
\end{abstract}

Keywords: Deterministic chaos; Lyapunov exponent; pseudo-periodic behavior; dynamical systems; dynamical disorder; time series.

\section{Introduction}

Many tools have been developed to study systems with chaotic behavior. Some of them are: the Lyapunov exponents [Wolf et al, 1985; Eekmann et al., 1986; Sano \& Sawada, 1985; Stoop \& Meier, 1988; Li \& Chen, 2004; Okushima, 2003]; Kolmogorov entropy [Kolmogorov, 1959; Bennetin et al., 1976; Grassberger \& Procaccia, 1983]; Poincaré section [Poincaré, 1892; Hénon, 1982]; fractal dimension [Mandelbrot, 1983; Badii \& Politi, 1986; Grassberger, 1987] and Hausdorff dimension
[Hausdorff, 1919; Rogers, 1970; Schleicher, 2005]. Such powerful methodologies are used to unveil the underlying complexity in nonlinear systems in order to understand how it can be modeled. Most real problems are nonlinear, hence there is a huge interest in having a set of tools to describe this kind of systems, to make realistic models and, of course, to make predictions. Many complex behavior, studied in chaotic equations, have already been observed in the real world. We could quote varied fields in which chaos has been found: Economics [Guegan, 2009], 
wind time series [Karakasidis \& Charakopoulos, 2009], computer processors [Halbiniak \& Jozwiak, 2007] and living cell populations [Laurent et al, 2010 .

Although the classical Nonlinear Dynamics Theory was set up some decades ago, new approaches have been proposed recently: the study of the topology of complex networks derived from time series for the characterization of the underlying dynamics [Zhang \& Small, 2006; Zhang et al., 2008; $\mathrm{Xu}$ et al, 2008); the use of the combination of a complexity measure and the Shannon entropy for distinguishing noise from chaos [Rosso et al, 2007]; the search for forbidden patterns in time series for detecting determinism [Amigo et al., 2008; Amigo et al, 2006; Carpi et al., 2010; Zanin, 2008]; the use of the Modified Sample Entropy as a regularity measure in time series [Xie et al., 2008; Xie et al., 2010]; the application of the $0-1$ test for finding chaos in deterministic systems [Gottwald \& Melbourne, 2009] and the extraction of the qualitative dynamical states in a system using Fuzzy c-Means Clustering [Shao et al, 2007].

Our purpose in this paper is to introduce two normalized indicators which combined together can be used as a complete method for characterizing nonlinear systems, distinguishing chaotic from stochastic behavior and measuring determinism. These indicators are the averaged "Perpendicular" and "Scalar" products which are measures of "Selfcorrelation" and "Dynamical order" respectively, concepts conveniently defined in the paper, always in the context of an orbit evolution within a proper space state.

The first hypothesis in this work is that the combination of the averaged "Perpendicular" and "Scalar" products, calculated over orbits derived from only one dimension, is enough to characterize the whole system. Hence, for m-dimensional systems, it would be enough to generate orbits from one of its variables. The second hypothesis is that the self-correlation, measured with the averaged "Perpendicular" product, provides the basis for modeling the system with equations in differences.

We present two different applications. The first one consists in a complete characterization of a system formed by two coupled nonlinear maps, that present a rich behavior which ranges from pseudo-periodicity to chaoticity. The second one consists in the successful modeling of two experimental time series belonging to a telephony network. Some important conclusions are derived from these applications: the "Perpendicular" product is a refinement of the "Mutual information", after the latter reaches its first minimum, and the "Scalar" product can work as a complement of the "Lyapunov exponent". Furthermore, some strong evidence of dimension reduction are provided for both, discrete and continuous systems. The "Perpendicular" product spectra are shown to be coupled for the whole variables which implies that it is possible to characterize the overall dynamics by the study on just one variable.

The scope of the method covers three different fields: general nonlinear systems, chaotic equations and experimental time series. In general, the first purpose will be to characterize the system. The second one will be to use the obtained information to model such system with equations in differences. Both, characterization and modeling are addressed in this work.

The paper is organized as follows. We present the methodology in Sec. 2, introducing three important definitions: averaged "Scalar" product, averaged "Perpendicular" product and "Escape velocity". We present an application to coupled nonlinear equations in Sec. 3. The relationship between "Dynamical order" and scale is presented in Sec. 4. In Sec. 5 we compare the averaged "Perpendicular" and "Scalar" products with the "Mutual information" and the "Lyapunoy exponent.". The dimension reduction is also addressed in this section. We present an application to experimental time series modeling in Sec. 6. Finally, we conclude the work in Sec. 7.

\section{Method}

Let us consider a generic $n$-dimensional chaotic system $\mathbf{X}(t, \omega)$ with $\eta$ parameters $\omega_{j}$, so that the system under consideration is of the general form

$$
\mathbf{X}\left(t, \omega_{1}, \ldots, \omega_{\eta}\right)=\left(X_{1}(t, \omega), \ldots, X_{n}(t, \omega)\right) .
$$

Fixing the initial conditions

$$
\mathbf{X}=\left(X_{1}^{0}, \ldots, X_{n}^{0}\right),
$$

and the values of the parameters vector $\boldsymbol{\omega}$

$$
\boldsymbol{\omega}=\left(\theta_{1}, \ldots, \theta_{\eta}\right)
$$

one may choose one of the variables $X_{j}$ and obtain a. time series

$$
X_{j}(t, \omega)=X_{j}(\Delta t, \omega), \ldots, X_{j}(N \Delta t, \omega),
$$


where $\Delta t$ is the sampling period for a continuous system. Its value is $\Delta t=1$ in the discrete case.

$X_{j}$ will be the object under study. The averaged "Scalar" $(p)$ and "Perpendicular" $\left(p_{\perp}\right)$ products are new indicators to measure dynamical order and self-correlation over a time series. The first one has been defined previously by other authors [Huerta et al, 1994], although we use it here in a. novel way. The second one is defined in this work for the first time. In order to calculate these indicators, the time series must be represented in an $m$-dimensional state space. For that purpose, it is necessary to fix two parameters: the dimension of the space $(m)$ and the time delay $(\tau)$. The dimension has the traditional meaning for mathematical spaces and the time delay is a natural number which signifies the relative position between two values in the time series. When the time series has "memory" and the value of $(\tau)$ matches with such memory, the hidden dynamic emerges from the state space.

For simplicity, in this work we will use $m=2$. The use of higher values of $m$ implies more computation time but it does not introduce further implications. We use this value without detriment to the general result.

\subsection{Averaged scalar product ( $p)$}

Consider a general time series derived from one of the variables of a chaotic system

$$
x(t)=x(1), x(2), x(3), \ldots, x(N) .
$$

For each value of $\tau$ a two-dimensional vector is obtained from the scalar series, forming a state for each value of $t$

$$
\mathbf{x}(t)=(x(t), x(t-\tau)) .
$$

The states evolution can be expressed as

$$
\begin{aligned}
\mathbf{x}(\tau & +1), \ldots, \mathbf{x}(t) \\
& =(x(\tau+1), x(1)), \ldots,(x(t), x(t-\tau)) .
\end{aligned}
$$

The flow vector $z(t)$, associated with each state $\mathbf{x}(t)$, is defined as

$$
\mathbf{z}(t)=\frac{\mathbf{x}(t+1)-\mathbf{x}(t)}{\|\mathbf{x}(t+1)-\mathbf{x}(t)\|}
$$

It is an unitary vector which points to the direction of the motion from state $x(t)$ to the next one $\mathrm{x}(t+1)$. Hence, $\mathrm{z}(t)$ shapes the trajectory along the states in space.
The "averaged flow vector" $\mathbf{V}(t)$ is calculated by considering $\mathbf{x}(t)$ as the center of a circle with radius $R$ and evaluating the quantity

$$
\mathbf{V}(t)=\frac{1}{N_{\xi}} \sum_{\xi \in \Omega} \mathbf{z}(\xi)
$$

where $\xi \in \Omega$ if $\|\mathbf{x}(\xi)-\mathbf{x}(t)\| \leq R$ and $N_{\xi}$ is the total number of points belonging to the inner circle.

At this point, it is worth clarifying two very important aspects:

Value of $\boldsymbol{R}$ : It is critical to choose a right value and it depends on the numerical series under study. If $R$ is too small, there will be very few points within every circle, hence $\mathbf{V}(t)$ will not be averaged enough. On the other hand, the average must have a local nature in the vicinity of every point of the space. If $R$ is too big rather a global average is obtained, since most points are in every circle. It is necessary to adjust the value of $R$ so that there are neither too many nor too few points within each circle. That value will be related to the states density in the space.

Dynamic meaning of $\mathrm{V}(\boldsymbol{t})$ : This vector represents the average dynamical evolution in the vicinity of every state. It points to the direction in which a local region moves to, on average. It is likely that the dynamical properties are not the same in all the space. In this case $\mathrm{V}(t)$ provides a view of the local dynamics.

Taking the latter aspects into account, the averaged "Scalar" product $(p)$ is calculated within all the state space by the following expression:

$$
p=\frac{1}{N-\tau-1} \sum_{t=\tau+1}^{N-1} \mathbf{z}(t) \mathbf{V}(t)
$$

where the product $\mathbf{z}(t) \mathbf{V}(t)$ provides a local measurement of dynamical order, since this product evaluates how parallel a single motion direction is with the averaged motion direction in its vicinity. The averaged "Scalar" product is obtained by adding all the local products along the space and dividing the result into the total number of states. If a space is very ordered, it will have a well defined vector $\mathbf{V}(t)$ for every point in space. In addition, $\mathbf{z}(t)$ will be parallel to $\mathbf{V}(t)$. As both vectors are unitary the local "Scalar" product will be close to 1. If these conditions are the same along the space the averaged product $(p)$ will be close to 1 as well. So, a state space which represents a time series is more 
ordered as fast as $p$ gets closer to 1 . In the same way, it is more random as $p$ gets closer to zero.

\subsection{Perpendicular product $\left(p_{\perp}\right)$}

When a periodic time series is represented in a state space with $\tau=T, T$ being the period, all the states $x(t)$ get restricted to the diagonal of the two-dimensional space. So, there are only two possible motion directions, both on the diagonal, which implies that there are only two possible values for any $\mathbf{z}(t)$

$$
\left(\frac{\sqrt{2}}{2} \mathbf{i}+\frac{\sqrt{2}}{2} \mathbf{j}\right) \quad \text { or } \quad-\left(\frac{\sqrt{2}}{2} \mathbf{i}+\frac{\sqrt{2}}{2} \mathbf{j}\right) .
$$

If $\tau \neq T$, the states are located out of the diagonal and a generic flow vector $\mathbf{z}(t)$ may have a huge variety of directions. With these considerations the "Perpendicular" product $p_{\perp}$ is defined as

$p_{\perp}=1-\frac{1}{N-\tau-1} \sum_{t=\tau+1}^{N-1}\left|\mathbf{z}(t)\left(-\frac{\sqrt{2}}{2} \mathbf{i}+\frac{\sqrt{2}}{2} \mathbf{j}\right)\right|$.

It is a sum of scalar products between flow vectors and a fixed vector, perpendicular to the diagonal. If $\tau=T, \mathbf{z}$ is on the diagonal $\forall t$, and all the products are equal to zero, resulting in $p_{\perp}=1$. On the contrary, if $\tau \neq T$ a certain number of flow vectors $\mathbf{z}(t)$ will be far from the diagonal and $\left(p_{\perp}\right)<1$.

$p_{\perp}$ may be used both, to test whether a value of $\tau$ is the period of a given time series and to make a self-correlation analysis in the case of nonperiodicity.

\subsection{Escape velocity $(m(\omega, \tau))$}

The "escape velocity" is

$$
m(\omega, \tau)=\frac{p_{\perp}(\tau, \omega+\Delta)-p_{\perp}(\tau, \omega)}{\Delta},
$$

where $\omega$ is one of the parameters in the system under study and $\Delta$ is the precision taken for the value $\omega$.

The system gains periodicity in $\tau$ when the "escape velocity" is positive, and loses periodicity when it is negative. A way to visualize this is by considering the system moving away from a periodic attractor $T\left(T^{\downarrow}\right)$ if $m(\omega, T)<0$ and getting closer $\left(T^{\dagger}\right)$ if $m(\omega, T)>0$. When the system remains at the same distance from the attractor $(\bar{T})$ $m(\omega, T)=0$. The possible values of $m$ are summarized as

$$
\left\{\begin{array}{l}
m(\omega, n T)<0 \Rightarrow T^{\downarrow} \\
m(\omega, n T)=0 \Rightarrow \bar{T} \\
m(\omega, n T)>0 \Rightarrow T^{\uparrow}
\end{array}\right.
$$

The "escape velocity" is a very useful indicator to calculate with precision the value of the parameter $\omega$ in which a bifurcation occurs. A detailed illustration of its use is provided in the next section.

\subsection{General methodology}

The general procedure to characterize chaotic systems involves the next four sequential steps:

(1) General scan with $p(\tau=1)$, for different values of $\omega_{j}, \forall j$.

(a) $p(\tau=1) \approx 1$ points out ordered areas.

(b) $p(\tau=1) \ll 1$ points out disordered areas.

(2) Correlation study with $p_{\perp}(\tau)$ in ordered areas, varying the value of $\tau$.

(a) $p_{\perp}(\tau)=1 \Rightarrow T=\tau$ (Periodic).

(b) $p_{\perp}(\tau) \approx 1 \Rightarrow T \approx \tau$ (Pseudo-periodic).

(3) Bifurcations study with $m\left(\omega_{j}, T\right)$ in periodic areas, varying $\omega_{j}$ slowly. A bifurcation takes place at $\omega_{j}^{0}$ if
(a) $\omega_{j}^{0}$ is a maximum for $m\left(\omega_{j}^{0}, T\right)$ and
(b) $\omega_{j}^{0}$ is a minimum for $m\left(\omega_{j}^{0}, T / 2\right)$.

(4) Disorder study with $p_{\perp}(\tau)$, in disordered areas.

(a) Detection of dominant correlations.

(i) $\tau_{0}$ is a dominant correlation if $p_{\perp}(\tau)$ has a. maximum at. $\tau=\tau_{0}$.

(b) Determination of chaotic or random nature.

(i) Chaotic system. $p(\tau, R)$ decreases slowly when increasing $R$.

(ii) Random system. $p(\tau, R)$ decreases quickly when increasing $R$.

Notice that the object under study may be a system of equations or a single experimental time series. The scheme presented above can confront both situations, although some of the steps do not apply in the characterization of 
an experimental time series. In this latter case, step (3) does not apply and (1) is only carried out once, since there are no system parameters $\omega_{j}$ to vary.

\section{Application 1. System of Two Coupled Nonlinear Maps}

Once the general method is introduced we proceed in this section to characterize a complex system consisting of two coupled nonlinear maps:

$$
\left\{\begin{array}{l}
x_{n}=\omega\left(3 y_{n-1}+1\right) x_{n-1}\left(1-x_{n-1}\right) \\
y_{n}=\omega\left(3 x_{n-1}+1\right) y_{n-1}\left(1-y_{n-1}\right)
\end{array}\right.
$$

where $\omega \in[0,1.19]$.

Two-dimensional coupled maps are strongly studied in many fields: chaos [López \& Pérez, 1991], complex patterns formation [López \& Fournier, 2003], cryptography [Fridrich, 1998], etc. We prove herein that these kind of systems can be characterized easily with the method proposed in the previous section. Despite the fact that the system is two-dimensional, a general description is possible by using only one dimension analysis. The study is done on the variable $x_{n}$ with initial conditions $x_{0}=0.3, y_{0}=0.76$. The calculations are carried out over orbits of 200 values, after discarding the first 10000 ones in order to eliminate the transitory evolution. The averaged "Scalar" products are calculated with a circle of radius $R=0.001$.

Figure 1 shows the bifurcation diagram for the variable $x$, along with its Lyapunow exponent, showing the complex behavior when $\omega>0.95$. Note that the Lyapunov exponent remains positive for most values of the parameter $\omega$ within the range shown in the figure.

\subsection{General scan}

A general scan of $x_{n}$ and $y_{n}$ has been carried out by calculating the "Scalar" product $p(\tau=1)$ for the range $[0,1.19]$ of parameter $\omega$. The results for $x_{n}$ are presented in Fig. 2. It shows that the system can be split into two different regions from a dynamical order point of view. The first region corresponds to a regular area with a boundary in $\omega=1.0295$. After this value of $\omega$, there is another region highly disordered. A detail of the regular zone is shown in the inset of Fig. 2 in order to observe its interesting structure.

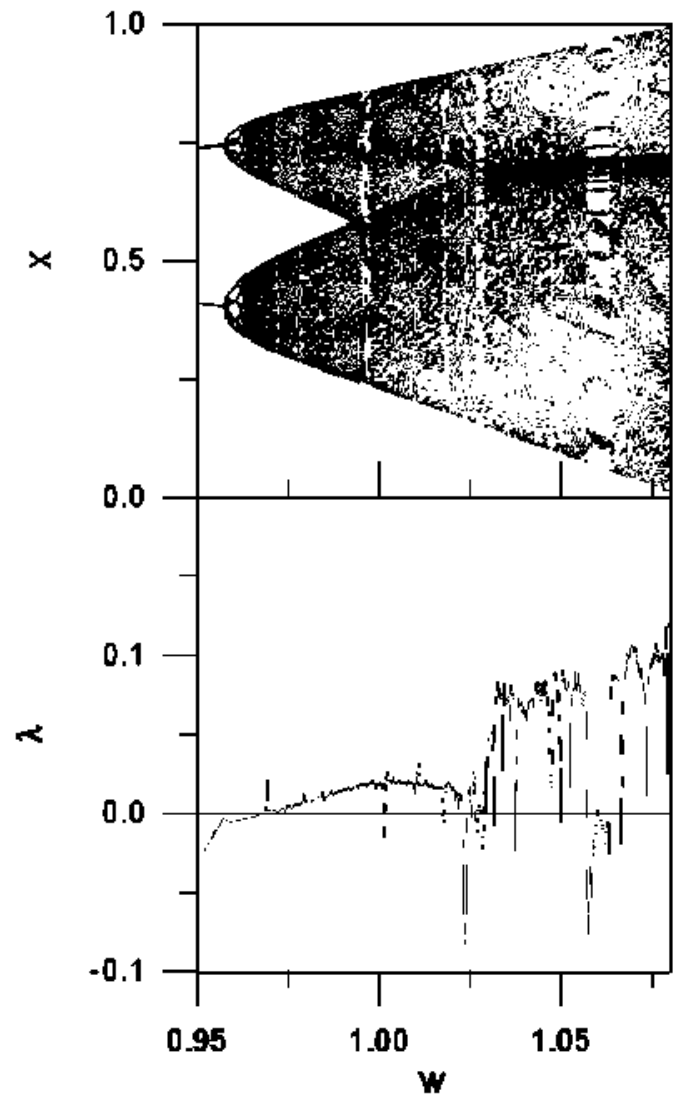

Fig. 1. Comparison of bifurcation diagram (top) and Lyapunov exponent (bottom) as a function of the parameter $\omega$ for the map defined by Eq. (15).

The results for the variable $y_{n}$ turned out to be the same as for $x_{n}$, which means that these coupled variables produce the same order scheme.

\subsubsection{Regular region. Period $T=2$}

The inset of Fig. 2 shows a small area with $p=1$ which is an indicator of periodicity. A correlation study on this area determines that it consists of periodic orbits with period $T=2$. Next, according to the correlation study, the system moves away from the period $T=2$ and comes into a pseudoregular behavior with intermittent regularity peaks. In Fig. 3 can be seen the calculation of the "escape velocity", $m(\omega, \tau=2)$, within the interval $\omega \in$ $[0.956,0.960]$. The results are quite clear and determine the exact value in which the system departs from a perfect periodicity, $\omega=0.95723$. Both, the maximum of the new period and the minimum of the former can be used to calculate a period duplication. However, in this system there is no period duplication, but a periodic state which changes into a pseudo-periodic one. So, in order to determine the 


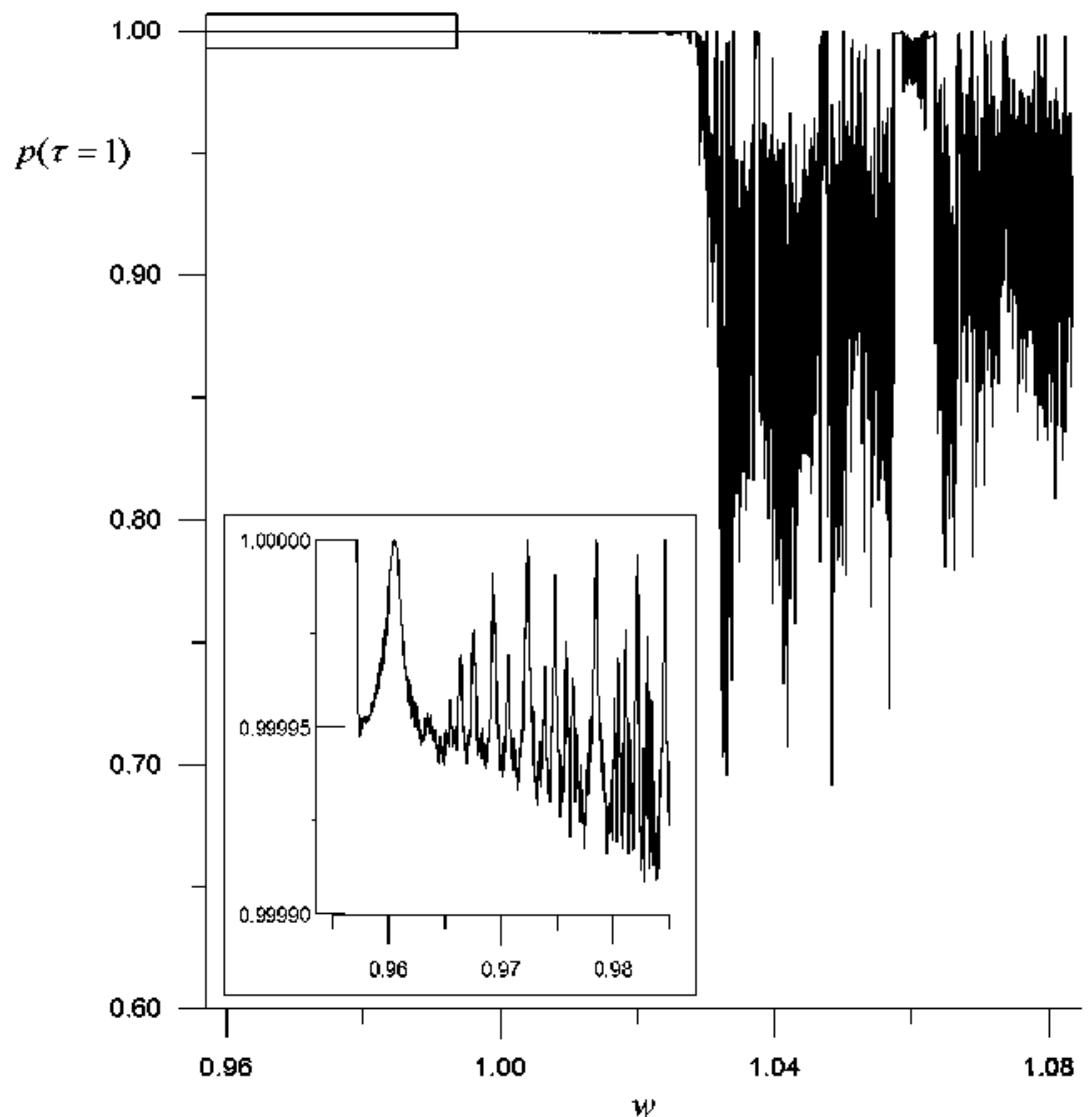

Fig. 2. General scan of $x_{n}$. Measurement of disorder by the "Scalar" product $p(1)$ as a function of $w$. The inset shows the structure of the regular area.

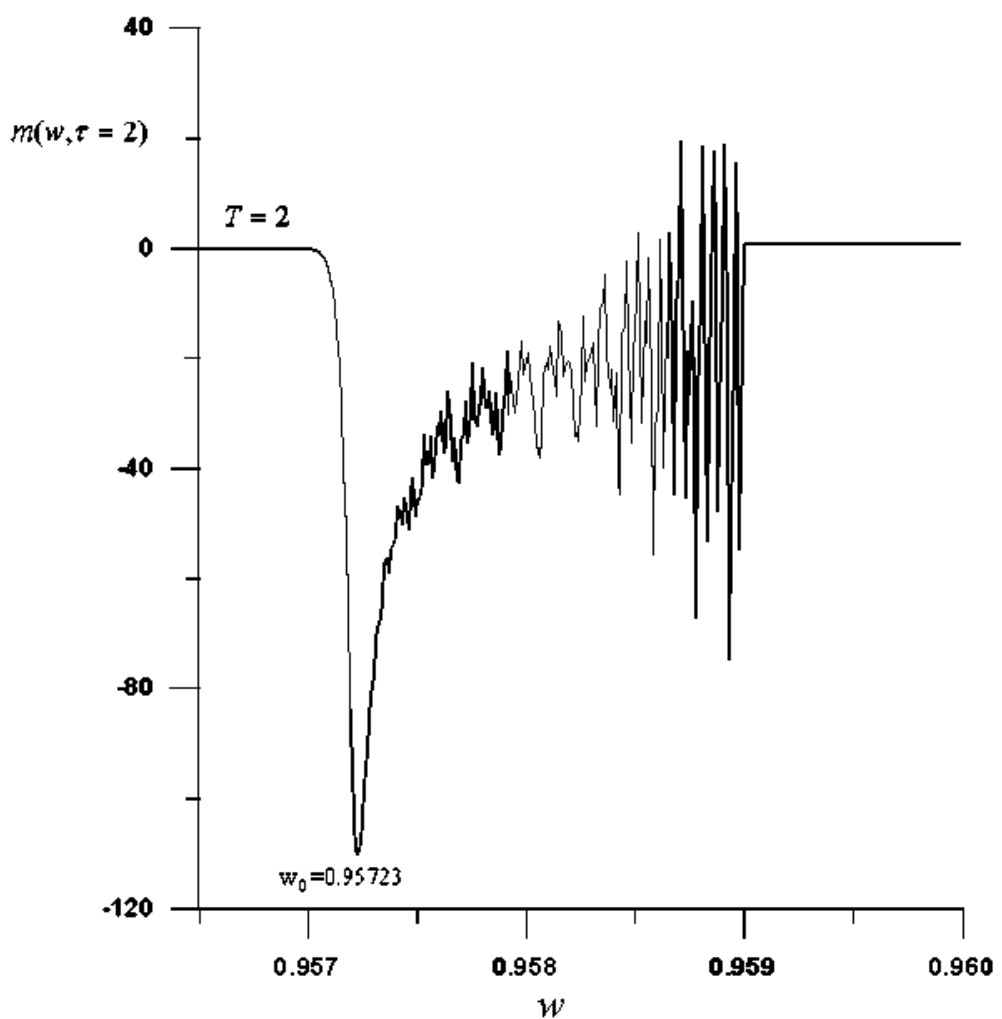

Fig. 3. Escape velocity. Calculation of the exact value of $w$ for which period $T=2$ is abandoned. 


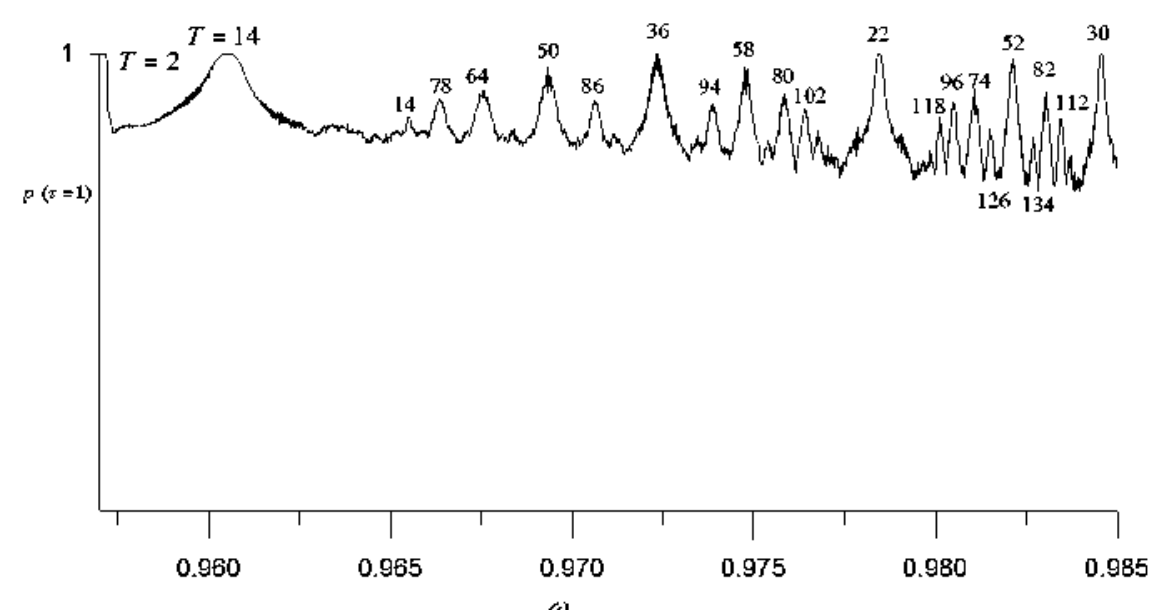

Fig. 4. Periodic and pseudo-periodic peaks in the interval $\omega \in[0.957,0.986]$. The numbers at the top of the peaks indicate the period of the corresponding orbits.

transition value between both states the minimum of $m(\omega, \tau=2)$ is used.

\subsubsection{Regular region. Pseudo-periodic behavior}

After departing from $T=2$, in $\omega=0.95723$, the system has an interesting structure. As can be seen in Fig. 2 the dynamical order $p(\omega, 1)$ decreases on average, but there are many shapely peaks which jump to the vicinity of $p(\omega, 1)=1$ indicating the presence of periodic or pseudoperiodic orbits. The study of the first peak is presented as an example of characterization. Once the general scan has detected the dynamical order close to $p=1$ for a given value of $\omega$, a correlation study must be

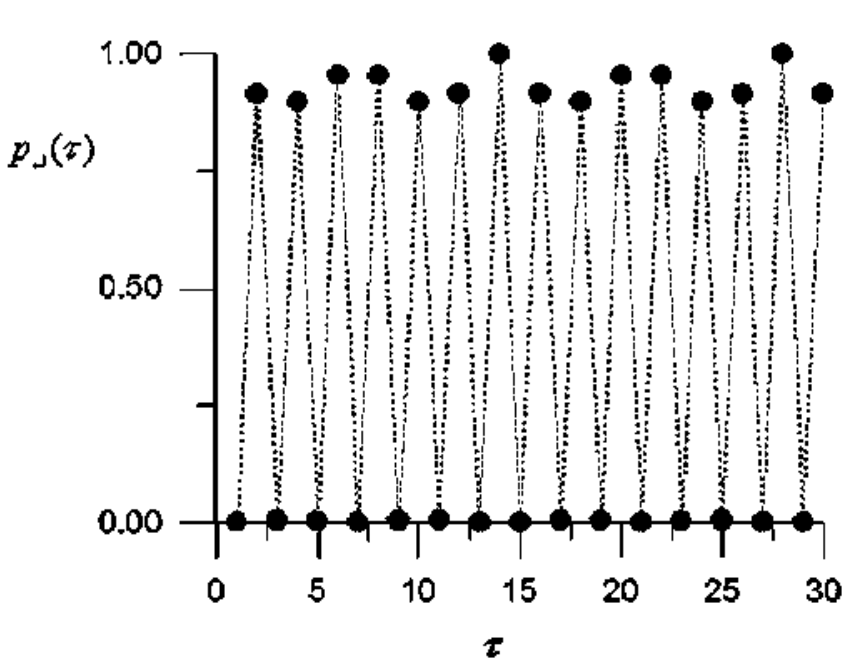

Fig. 5. Correlation study for $\omega=0.96048 . \tau=14$ and its multiples are dominant delays. carried out on such value. In the case of the first peak, the maximum value of $p(\omega, 1)$ is reached in $\omega=0.96048$. In other words, it is the value of $\omega$ at the top of the first peak in Fig. 4. Figure 5 shows the correlation study for this value. The predominant periods are $\tau=14$ and its multiples. The obtained value $p_{\perp}(0.96048,14)=0.9999<1$ indicates that it is not a perfect periodic orbit. In order to clarify whether there is a perfect periodic behavior in the vicinity of this value it is necessary to increase the precision. A calculation of $p_{\perp}(\omega, 14)$ was carried out within the interval $[0.96045,0.96060]$ with a precision of ten decimal places and the results are shown in Fig. 6. A periodic orbit is found in $\omega=0.9605236799$.

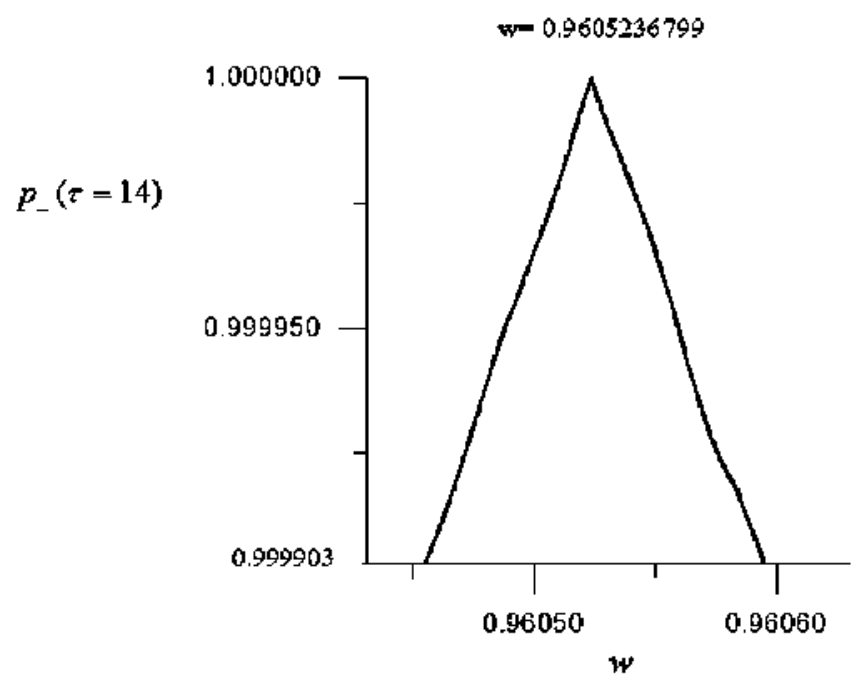

Fig. 6. "Perpendicular" product as a function of $\omega$. The top of the peak indicates a periodic orbit, while the values of $\omega$ at the right and left correspond to pseudo-periodic ones. 


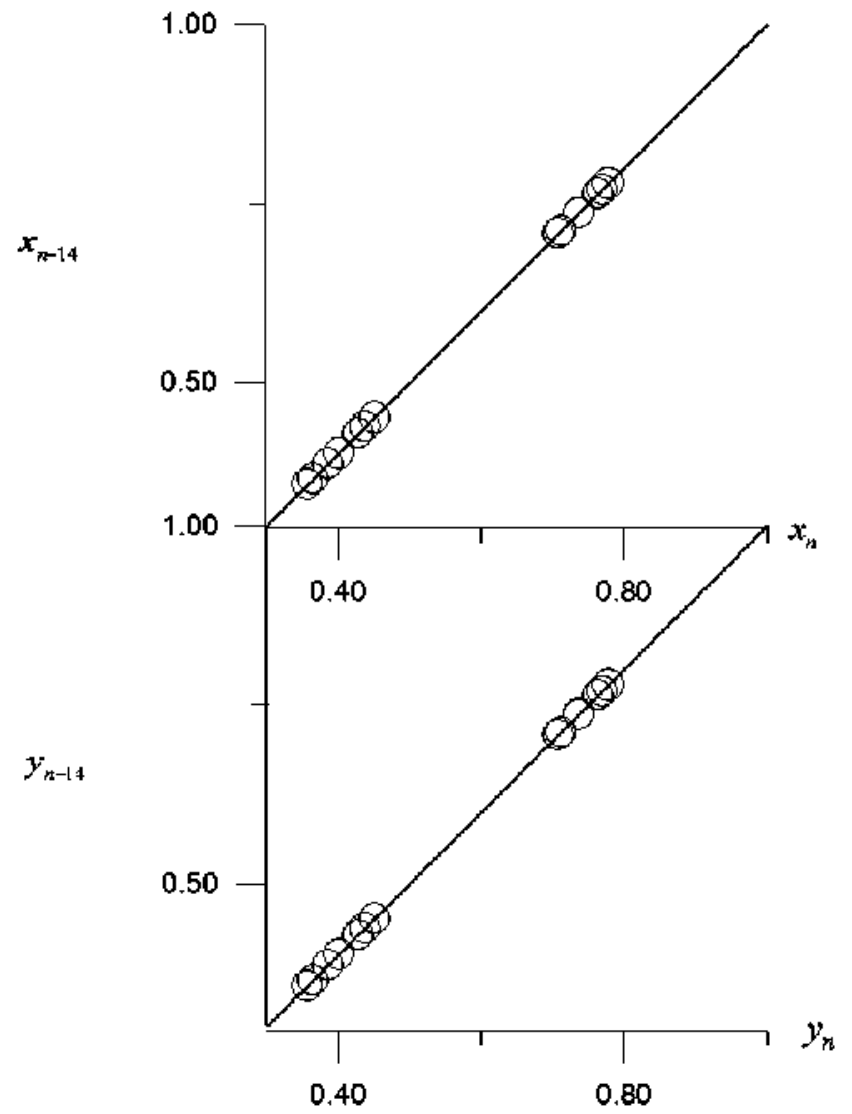

Fig. 7. Period $T=14$ corresponding to $w=0.9605236799$. State space for variables $x_{n}$ and $y_{n}$.

Hence, the system is periodic at this value and pseudo-periodic to the right and left. The approach from pseudo-periodicity to a perfect period $T=14$ is smooth and builds a curious shape, shown in

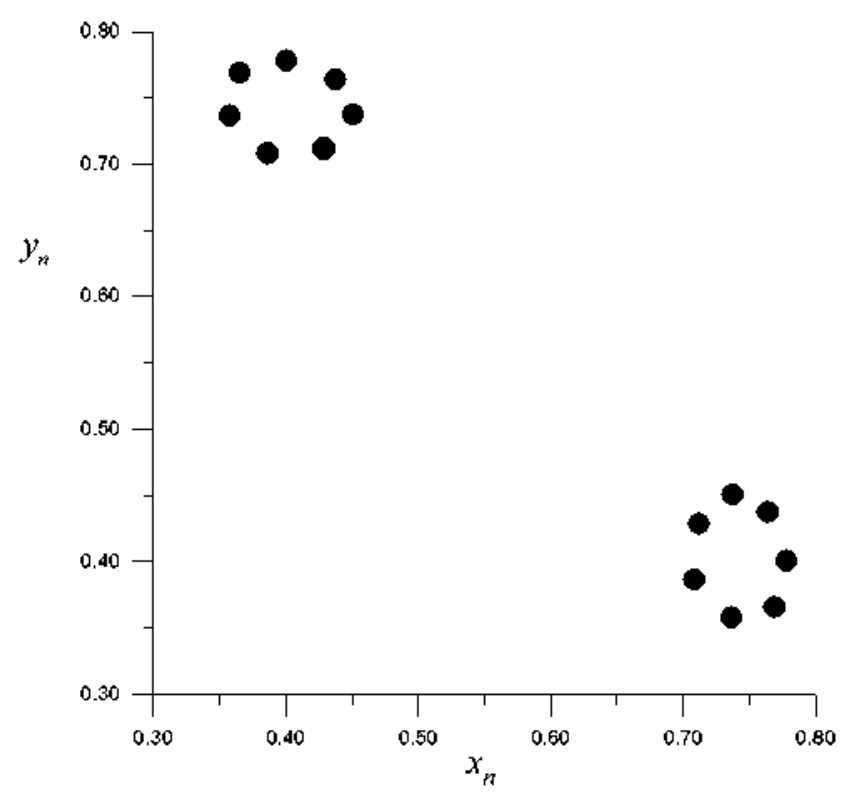

Fig. 8. Two-dimensional state space for $\omega=0.9605236799$
Fig. 6. Figure 7 shows the state space for variables, $x_{n}$ and $y_{n}$ and makes clear that both orbits are perfectly periodic. Two-dimensional state space can be visualized in Fig. 8. It is important to underline that a two-dimensional problem has been reduced to one dimension.

The previous characteristics have already been found in the remaining peaks. The corresponding periods are shown in Fig. 4, although only for some cases where the peak reaches perfect periodicity.

\subsubsection{Disordered region}

In order to prove that the general method can be used to characterize highly disordered systems, it is applied to an orbit located in the most disordered region of Fig. 2. Figure 9 shows the orbit for $\omega=1.04141$ and the results of the correlation study are presented in Fig. 10. The dominant period is $\tau=53$, both for variable $x_{n}$ and $y_{n}$. The value of $p_{\perp}(53)$ is 0.575702 for $x_{n}$ and 0.55661 for $y_{n}$, which indicates a low level of correlation. From Fig. 10, it follows that the correlation structure is very similar for $x_{n}$ and $y_{n}$. The state space for $x_{n}$ is shown in Fig. 11, where an attractor is observed. The two-dimensional state space is depicted in Fig. 12 where the details of the global attractor emerge. It is important to underline again that the dynamies of this two-dimensional system has been characterized using the one-dimensional method proposed

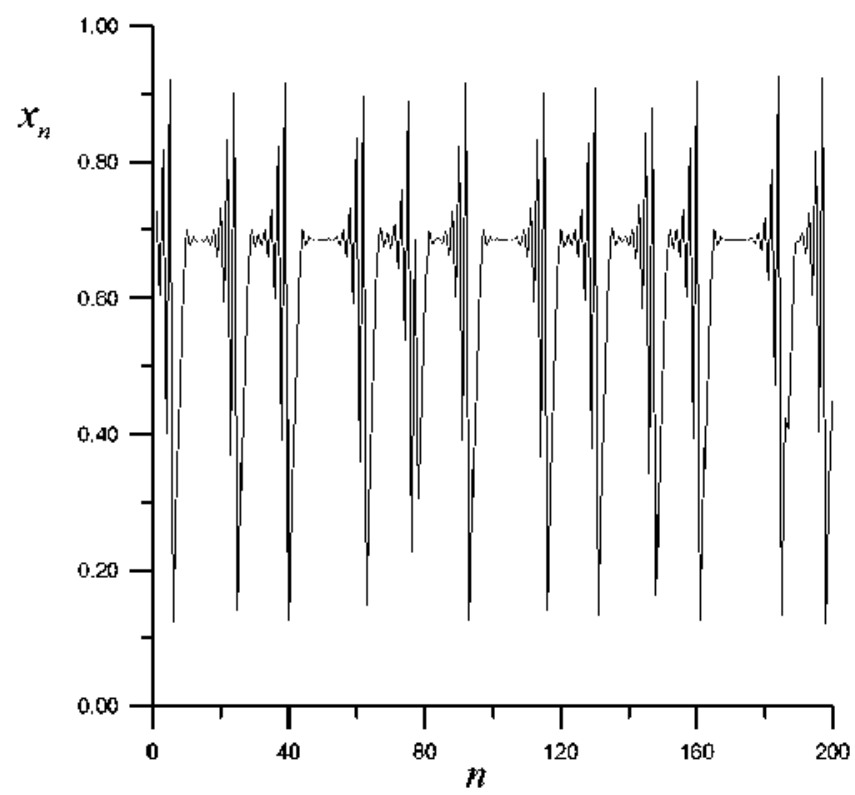

Fig. 9. Orbit in a disordered region of the system. $\omega=$ 1.04141 . 


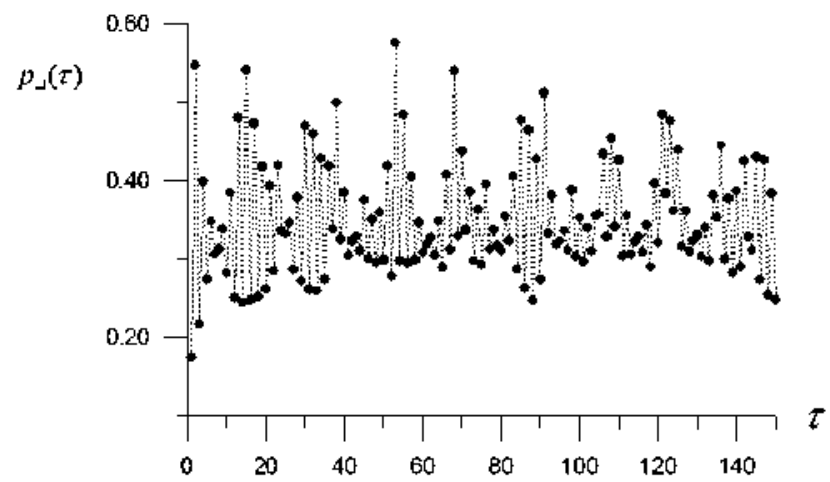

(a)

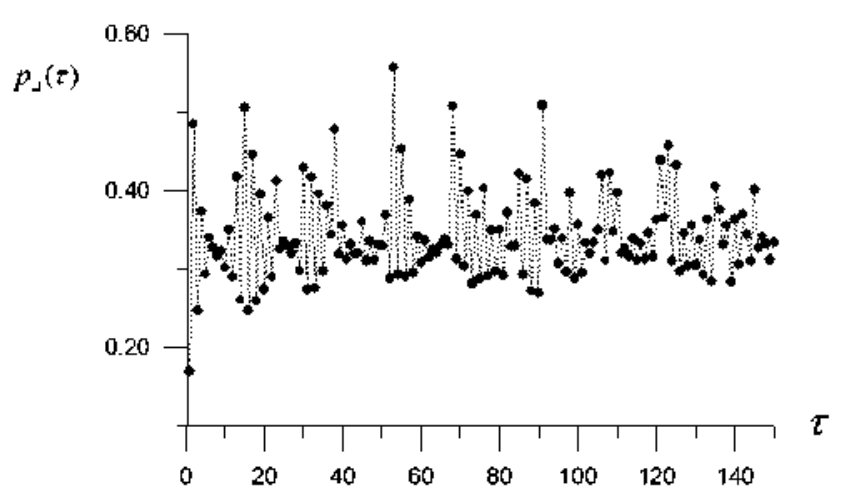

(b)

Fig. 10. Correlation study for $\omega=1.04141$. (a) Variable $x_{n}$, (b) variable $y_{n}$.

in this work. Every variable is obviously affected by the other and the information contained in any of them is enough to characterize the whole system.

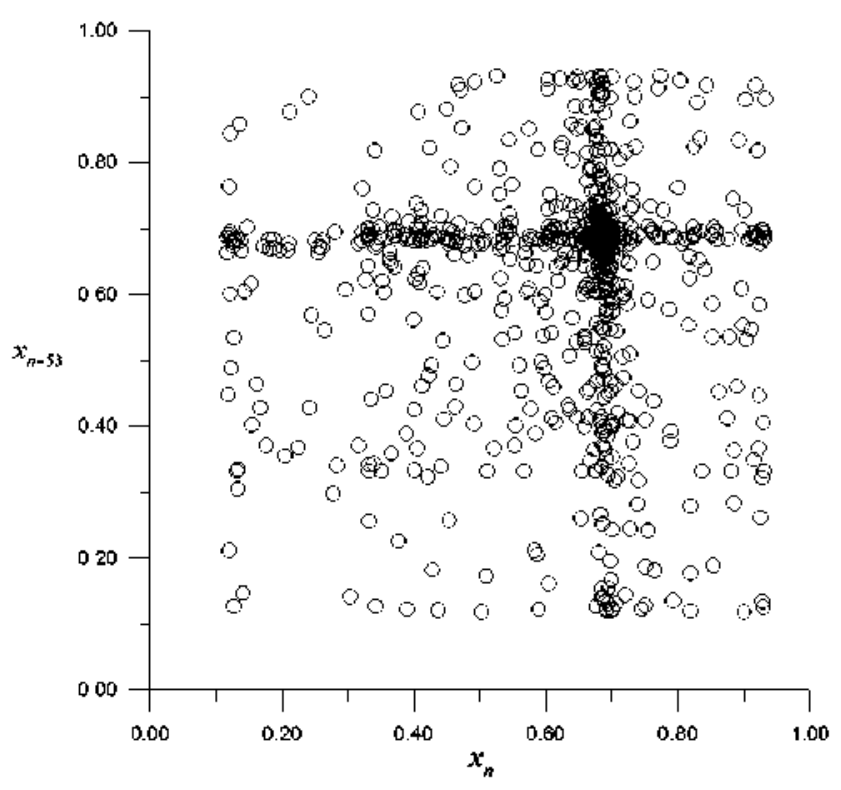

Fig. 11. State space for $\omega=1.04141$.

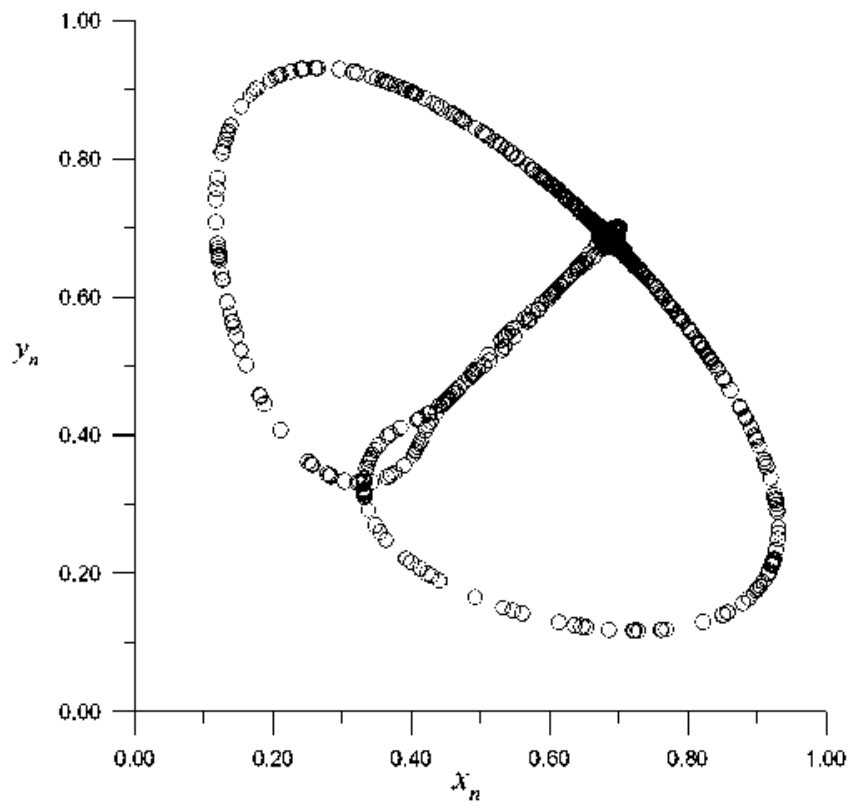

Fig. 12. Two-dimensional state space for $\omega=1.04141$.

\section{Disorder and Scale}

The variation rate of the "Dynamical order" with the scale is a characteristic of every system. The comparison of $p(\tau)$ for two different systems measures their different dynamical order at a certain scale $R$ and this is enough for most purposes. However, if this dynamical order needs to be studied in depth, a slow variation of $R$ must be done to cover the entire scale. In order to show how different this variation rate might be, a comparison between two extreme systems is done. Figure 13 compares a chaotic orbit with a random series and shows how the dynamical order depends on the scale $(R)$. Initially, the order drops very fast in the random series

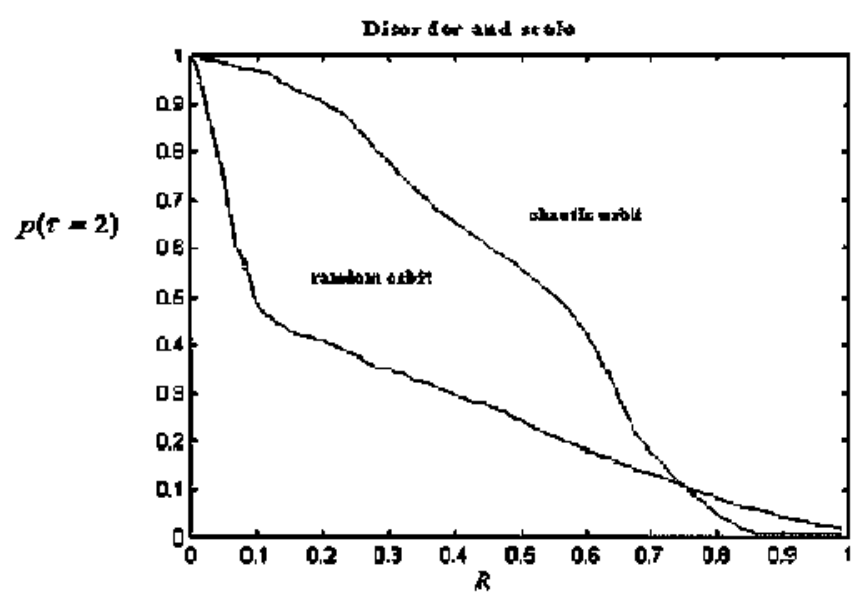

Fig. 13. Chaos versus randomness. Dynamical order decreases faster with $R$ in the case of randommess. 
whereas it is kept quite high in the chaotic orbit. They both decrease with the scale but much more gradually in the case of chaos. The reason can be found by analyzing the behavior of the flow vectors in every single circle with radius $R$. In a random time series, a single point can move towards any direction with the same probability. Hence, one may find a continuous range of flow vectors within the circle. When increasing the radius $R$ more points get included and the random nature of the new flow vectors makes $p$ smaller. In the presence of chaos, at a small scale, the flow vectors are quite parallel due to local dynamical order. When increasing $R$, the circles include neighboring points with other dynamical patterns, so $p$ decreases in every scale change, but accumulating less disorder than in the random series.

This result indicates that chaotic systems are very ordered from a "microscopie" point of view. However, at a "macroscopic" scale, the system seems to be random. The scale under consideration

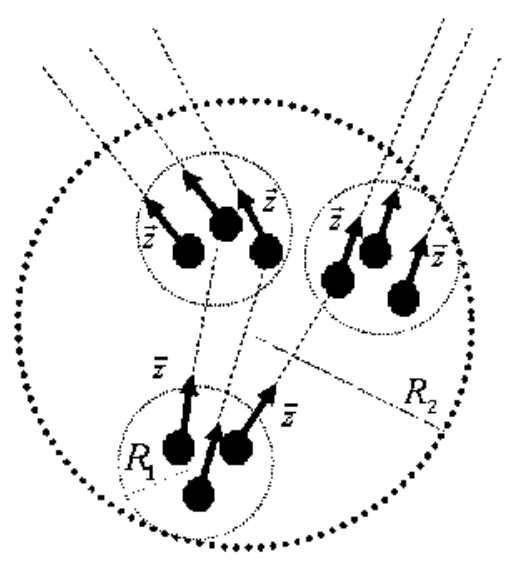

(a)

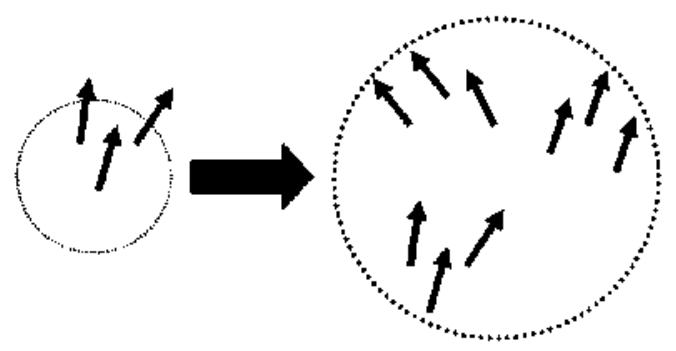

Light increase in disorder

(c) is very important when it comes to classifying a system as random or chaotic.

Figures 14(a) and 14(b) present some details on the directions taken by the flow vectors in chaotic and random state spaces. Figure 14(a) corresponds to a chaotic system. When a circle with a small radius $R_{1}$ is considered, the included states have similar dynamical characteristics. In other words, their flow vectors are really parallel. At this scale, the system is quite ordered. Increasing the scale by taking a greater radius $R_{2}$, the new included states have smooth differences in their flow vectors with those considered in the previous circle, and already present in the new one. Now, the same circle includes different behavior, so the internal disorder increases. A change of scale in a random system causes an increase as well in the dynamical disorder but the effect is much more abrupt. Figure 14(b) shows that the flow vectors are not parallel at the scale $R_{1}$. The system is not specially ordered at this scale, but its random nature is not already

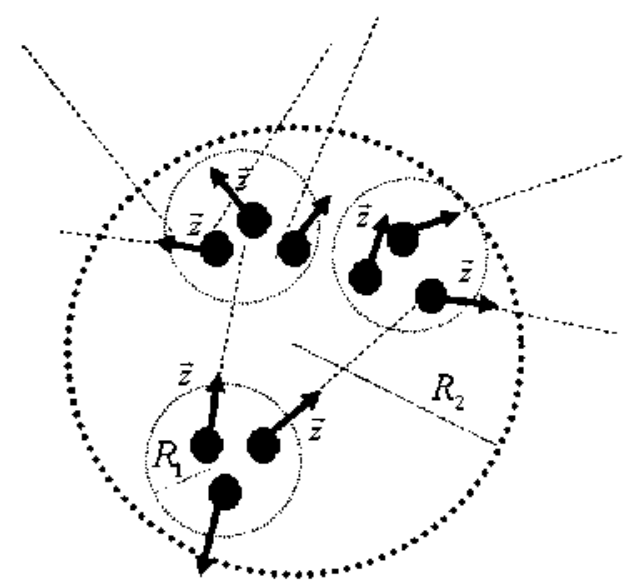

(b)

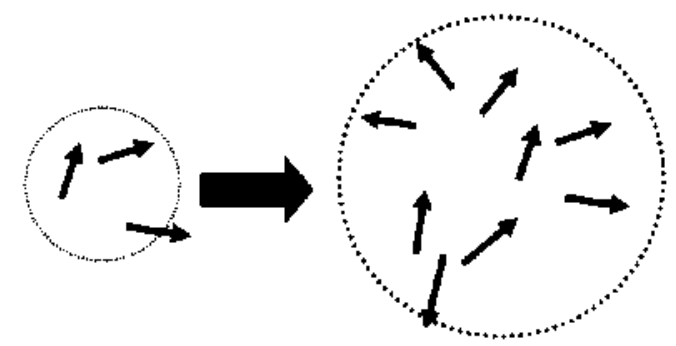

Strong increase in disorder

(d)

Fig. 14. Dynamical order as a function of the scale. (a) Flow vectors in a chaotic system. (b) Flow vectors in a random system. (c) Light increase in disorder for a chaotic system when increasing the scale. (d) Strong increase in disorder for a random system when increasing the scale. 
obvious since the amount of states in the circle is not enough to have a good statistical average. This random nature requires enough states in the circle to be obvious. The disorder increases considerably in the change of scale with $R_{2}$, since every incorporated group of states contributes randomly oriented flow vectors. So, the quantity of disorder which is incorporated only depends on the amount of new states included in the change of scale. However, in a. chaotic system the new states are groups of flow vectors with local homogeneity, so the disorder incorporated depends on the amount of new states and the orientation of the local clusters. Figures 14(c) and $14(\mathrm{~d})$ show that the entry of the same number of states into a circle, in a change of scale, causes a larger increase of disorder for a random system.

In general, the increased rate of disorder depends on the specific system, it will be smooth in the case of regular determinism, strong in chaotic systems and extremely strong in the case of random processes. The comparison of the evolution of $p(\tau, R)$ for two general series is a measure of how different they are from a dynamical order point of view.

\section{Lyapunov Exponent, Mutual Information and Dimension Reduction}

The method detailed in the previous sections is based on two basic indicators: "Perpendicular" and "Scalar" averaged products. The purpose of this section is to frame these indicators within the context of the current nonlinear science by establishing a relation among them and three of the most important measures available of late "Lyapunov exponent", "Mutual information" and "Dimension".

\subsection{Lyapunov exponent}

The calculation of the Lyapunov exponent [Wolf et al., 1985; Eckmann et al., 1986; Sano \& Sawada, 1985; Stoop \& Meier, 1988; Li \& Chen, 2004; Okushima, 2003] for a variable provides clear information about the sensitivity to initial conditions, which is also a measure of the presence of chaos. Positive values of the exponent imply chaotic behavior where near trajectories diverge from each other, whereas negative values imply that such trajectories converge together. The strength of this divergence or convergence depends on the absolute value of the Lyapunov exponent.
Now, it is interesting to establish if the averaged "Scalar" product can supply similar information or even supplement the Lyapunov exponent in some ways. Regarding the nature of the information provided by both measures, it can be proved that this information is quite similar. While the "Lyapunov exponent" is calculated over a really long evolution of two near trajectories, the averaged "Scalar" product calculates the divergence of multiple trajectories for a short time evolution within a small volume of the state space. When calculating scalar products among flow vectors belonging to near points, the result is a measure of divergence only for one time step. That is to say, the measure does not average over a complete trajectory for two initial conditions but over multiple initial conditions for only one time unit. As this local calculation is carried out within the whole space, the final result can be assumed as a global divergence rate.

On the other hand the averaged "Scalar" product has two interesting characteristics which supplement the Lyapunov exponent. The first characteristic is that it is a normalized indicator of dynamical order, which provides a quick impression of the amount of underlying order in a system. For instance, if a system in an ordered regime with $p=1$ is suddenly affected by external noise and the next measure comes to $p=0.95$, one can state that the external noise has introduced a $5 \%$ of disorder according to an absolute scale, since $p=1$ is the maximum order state $(100 \%)$. The second characteristic is that $p$ can distinguish small changes in the dynamics of a system due to its high sensitivity to dynamical order changes. Averaged "Scalar" product and Lyapunov exponent are compared for the logistic map in Fig. 15. Figure 15(a) shows how $p$ distinguishes clearly the areas with positive Lyapunov exponent from those with negative ones. What is more, $p$ goes beyond the limits of the "Lyapunov exponent", since it reaches higher precision when differentiating close dynamical states. Figure 15(b) shows how $p$ reveals a region with a variety of dynamical states where the Lyapunov exponent only detects a rather flat behavior. An analysis of the states, marked by an arrow in Fig. 15, is shown in Fig. 16. Every state corresponds to a value of $\omega$ in the logistic map. Both have similar Lyapunov exponents but they are dynamically quite different. The decrease in the value of $p$ with $R$ is faster for $\omega=3.688$ which indicates significantly more disorder in this state (see Sec. 4). 


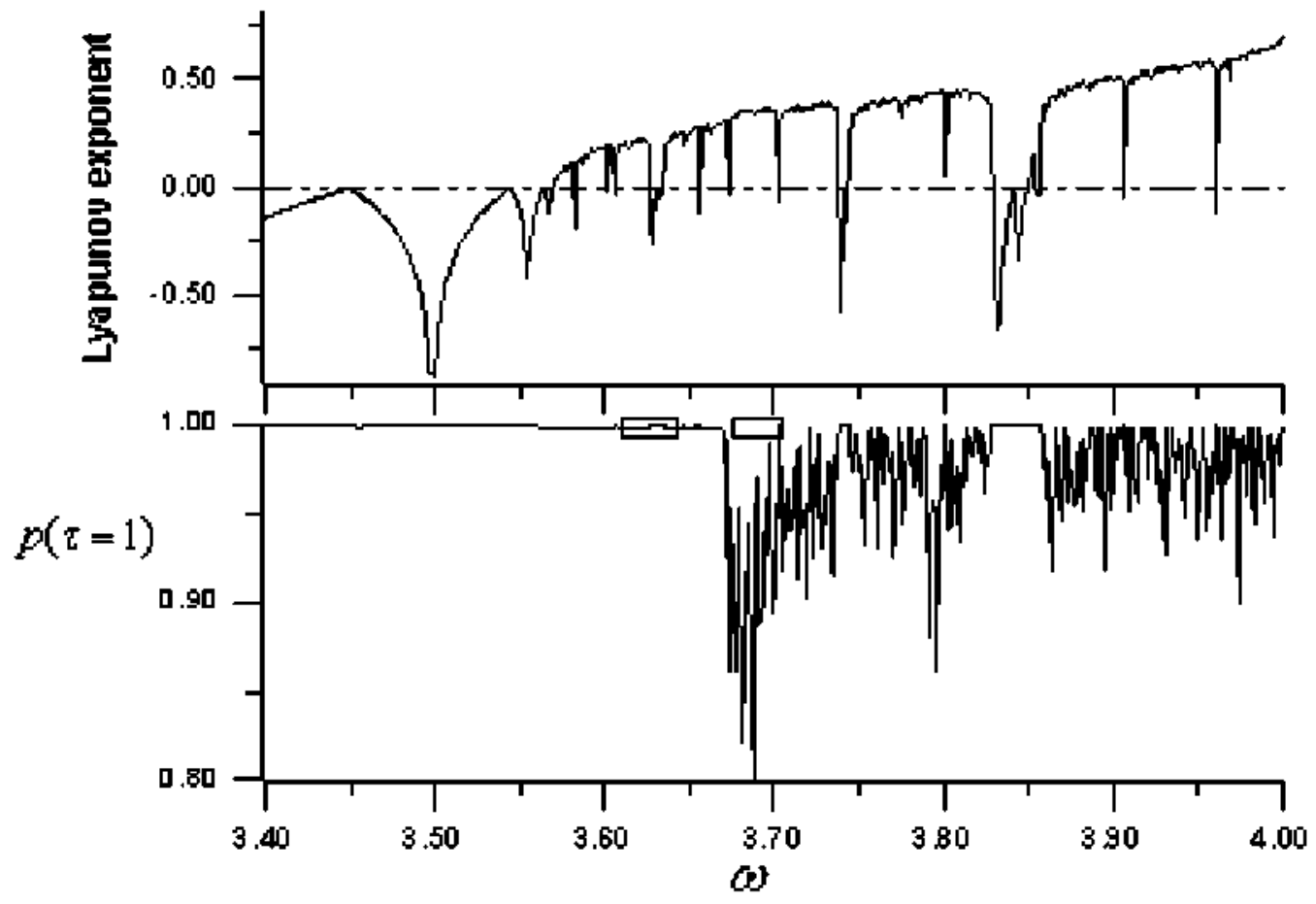

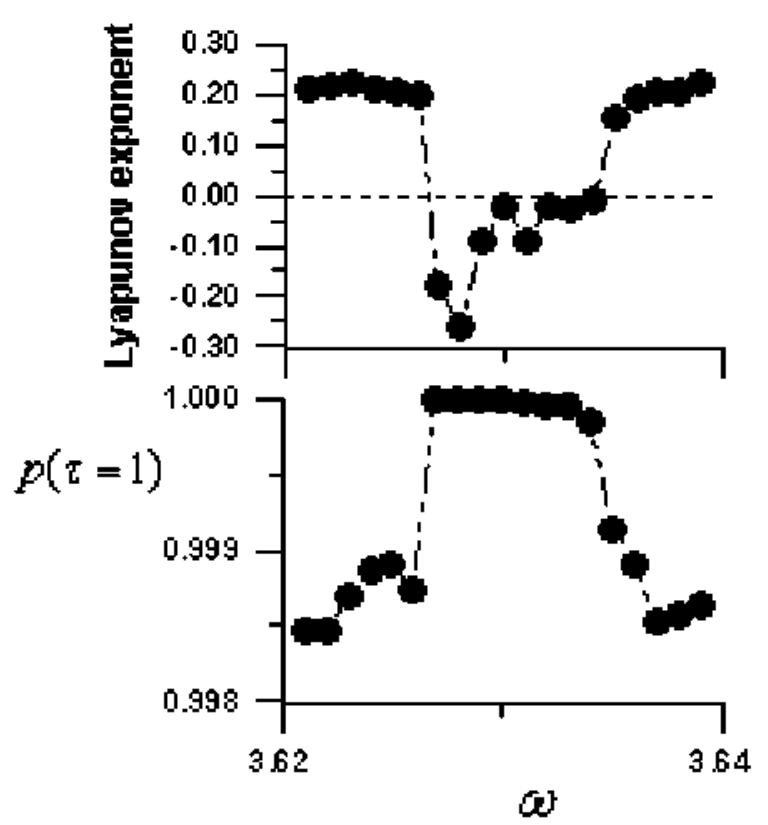

(a)

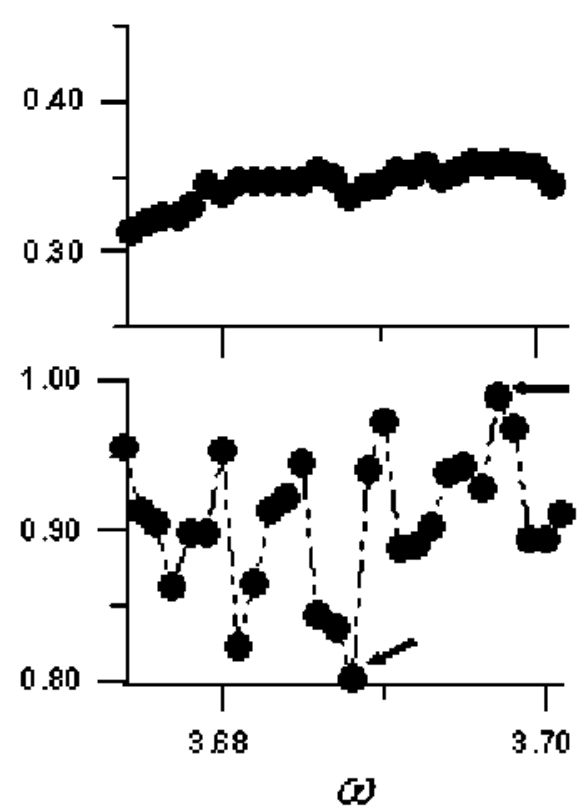

(b)

Fig. 15. Top: Lyapunov exponent versus averaged "Scalar" product for logistic map. Bottom: Expanded details of the ordered area (a) and disordered area (b).

\subsection{Mutual information}

Mutual information (MI) [Cellucci \& Albano, 2005; Khan et al, 2007; Shannon \& Weaver, 1949] is a measure widely used in multiple applications as an indicator of nonlinear correlation between two variables. In the field of time series the measure of interest is the "Auto Mutual Information" (AMI) consisting of the calculation of MI between a time series and itself delayed $\tau$ time units [Fraser \& Swinney, 1986]. A similar approach is followed in this work - the measurement of mutual correlation in a series by the averaged "Perpendicular" product $\left(p_{\perp}\right)$. In order to compare AMI and $p_{\perp}$, both measures have been calculated for two chaotic orbits 


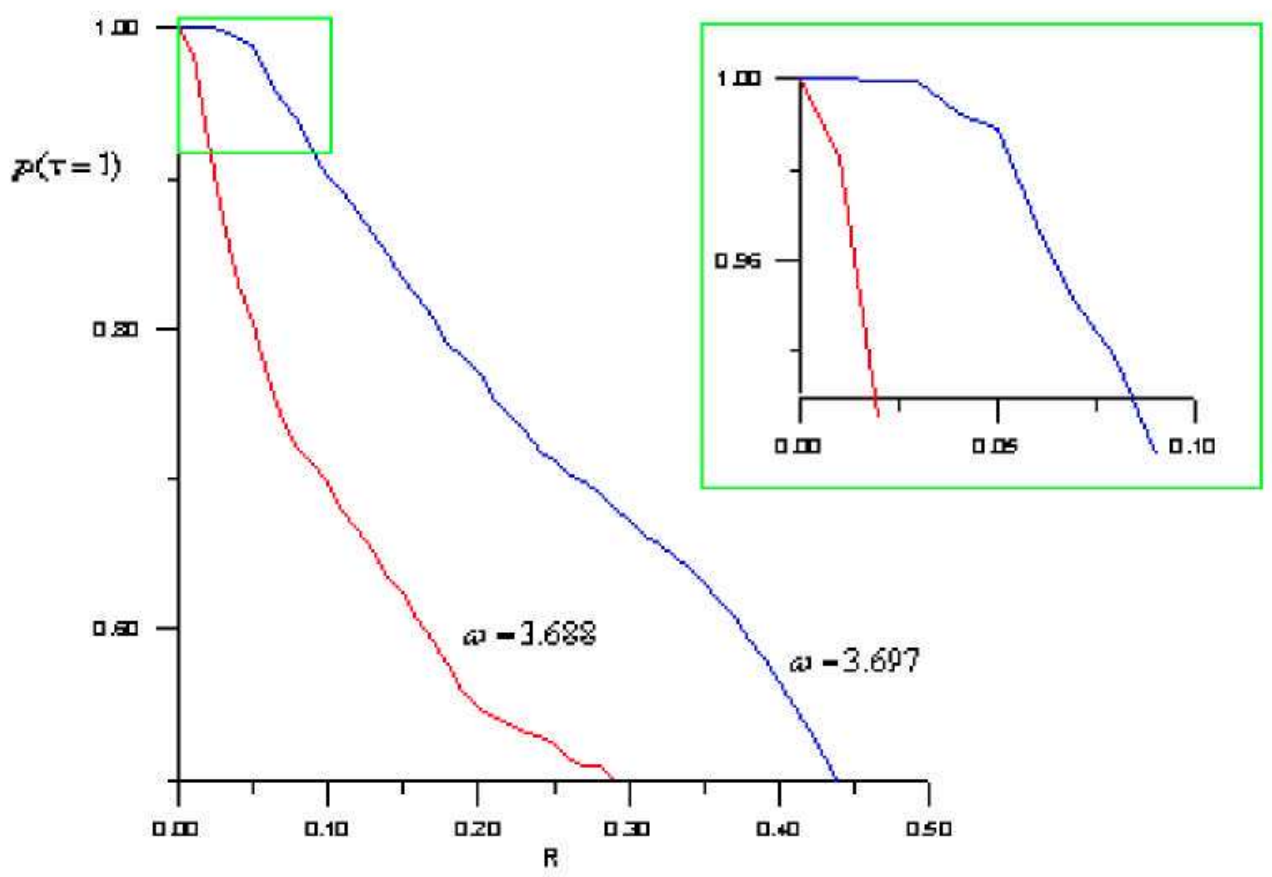

Fig. 16. Evolution of $p$ as a function of $R$ for the two states marked by an arrow in Fig. 15(b).
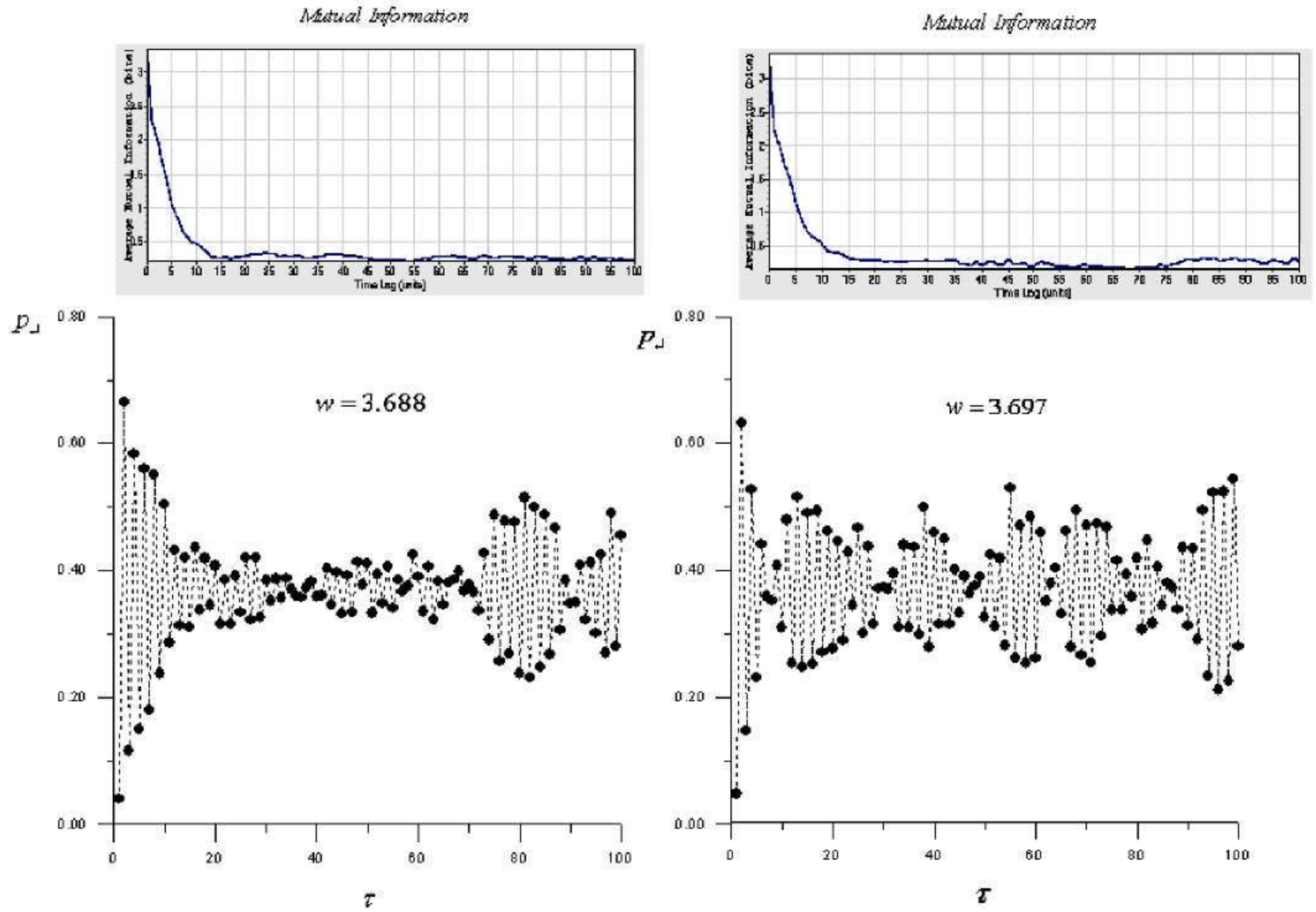

Fig. 17. AMI and $p_{\perp}$ for chaotic orbits of the Logistic map. 
belonging to the Logistic map and two experimental time series. The results for the chaotic orbits are shown in Fig. 17. It can be observed in the upper graphs that AMI provides the decrease in nonlinear correlation as a function of the delay $\tau$, without expressing any additional information after reaching the first minimum. However, in the lower graphs, $p_{\perp}$ shows a richer structure of auto correlation along all the values of $\tau$. This structure is a deep characteristic of the series which can be used for modeling. The modeling of experimental time series by using its correlation study is addressed in the next section.

Two experimental time series are shown in Fig. 22 and a comparison of the results of $p_{\perp}$ and AMI for those time series is presented in Fig. 18. They correspond to provincial and international telephony traffic measured throughout a year. The details of the nature of these time series are commented on in the next section. Both series are pseudo-periodic although the one shown in Fig. 18(b) is much more complex, which hides its underlying pseudo-periodicity. The calculation of AMI for the provincial traffic series provides a clear

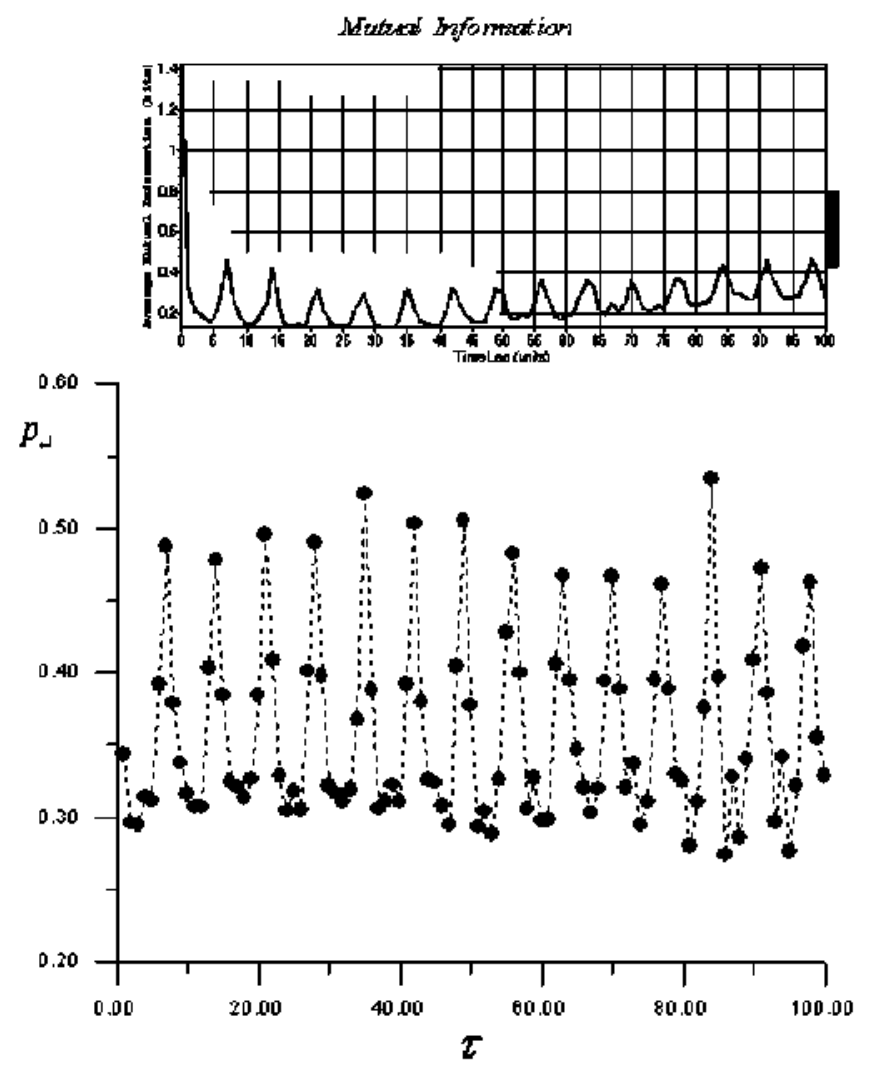

(a) result of pseudo-periodicity with period $T=7$. The same conclusion is obtained by the calculation of $p_{\perp}$. Nevertheless, in the case of international traffic AMI does not provide any special structure of correlation, whereas $p_{\perp}$ shows a clear pseudo-periodicity, again with period $T=7$. As it will be shown in the next section, the international traffic series can be modeled satisfactorily based on the information derived from the calculation of $p_{\perp}$.

\subsection{Dimension reduction}

In this section, we present some arguments and strong evidence which point out that the introduced method can reduce the study of an $m$-dimensional system to the study of only one of its dimensions. This statement claims not to be applicable only to symmetric systems, like the one studied in (Sec. 3), but to general m-dimensional systems. Both variables, belonging to the coupled maps system, have practically the same correlation study as can be seen in Fig. 10. Initially it might be assumed that this result is derived from the symmetric form of its mathematical expression, however similar results

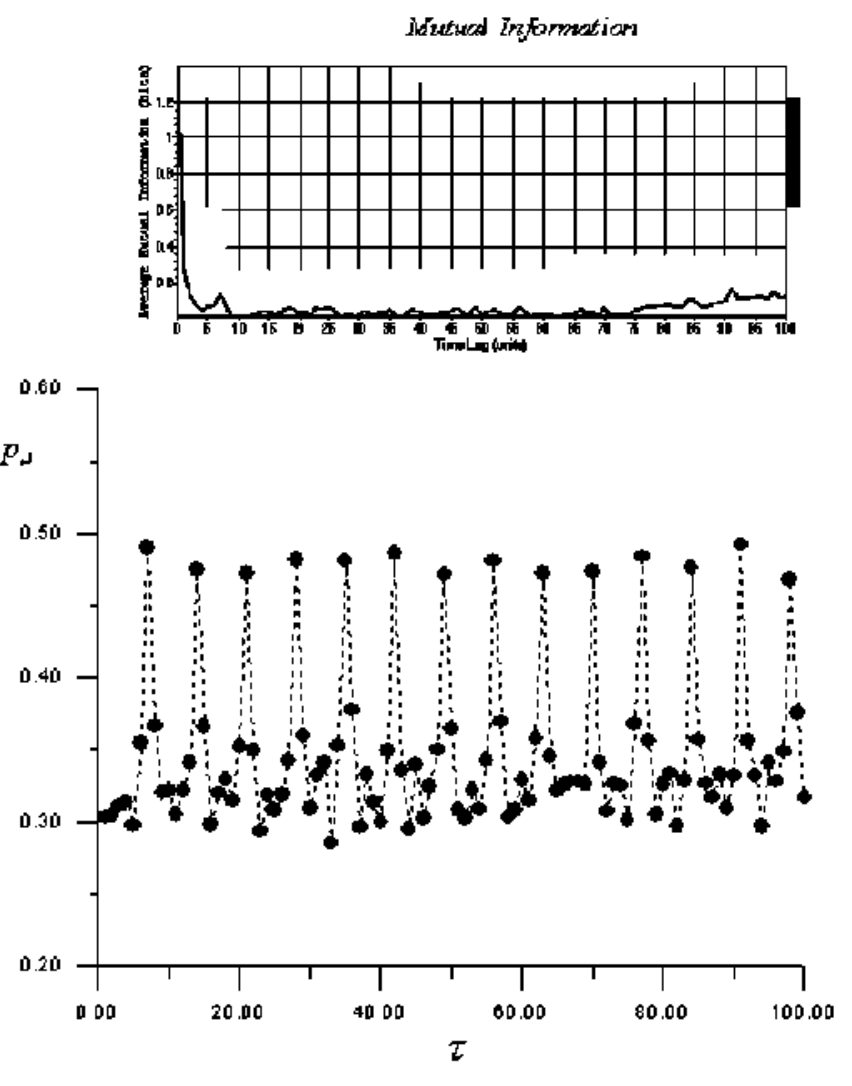

(b)

Fig. 18. AMI and $p_{\perp}$ for telephony traffic. (a) Provincial traffic. (b) International traffic. 


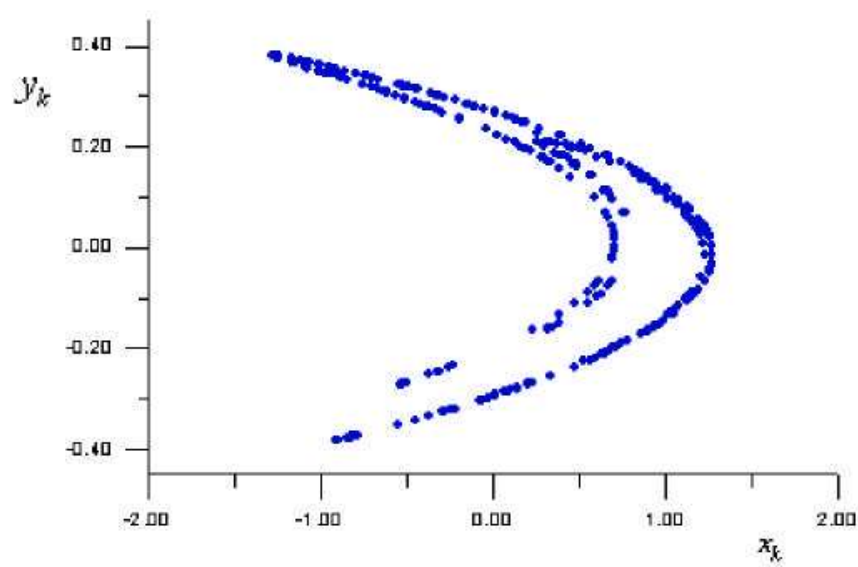

(a)

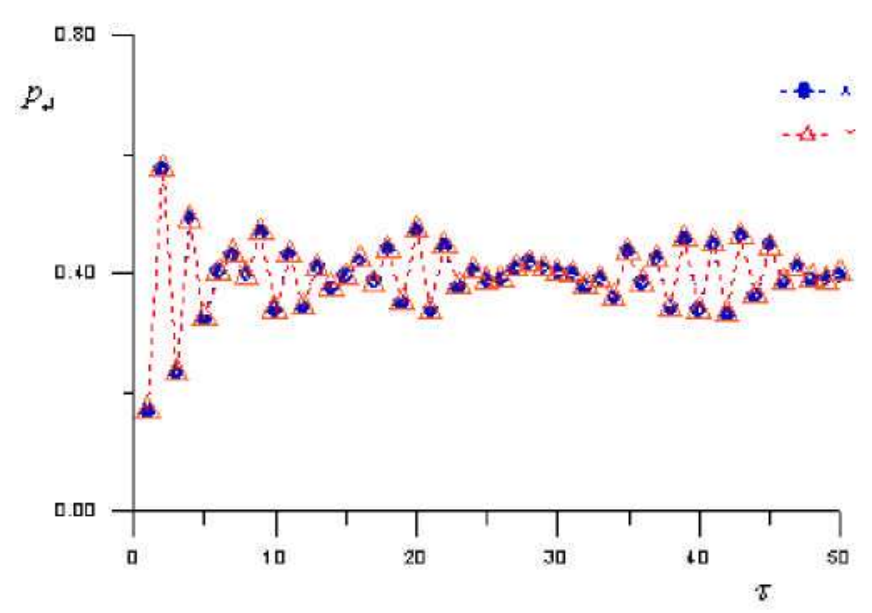

(b)

Fig. 19. Henon attractor (a). Correlation study (b).

have been found for three well known chaotic attractors: Henon, Lorenz and Rossler. These results are shown in Figs. 19-21.

Henon attractor [Hénon, 1976] has the following expression

$$
\begin{aligned}
& x_{n+1}=y_{k}+1-a x_{k}^{2} \\
& y_{k+1}=b x_{n} .
\end{aligned}
$$

The relationship between both variables cause again an identical structure in Fig. 19, without a special form in the mathematical expression of the system.

In order to extend the study to continuous systems with dimension three, two well known

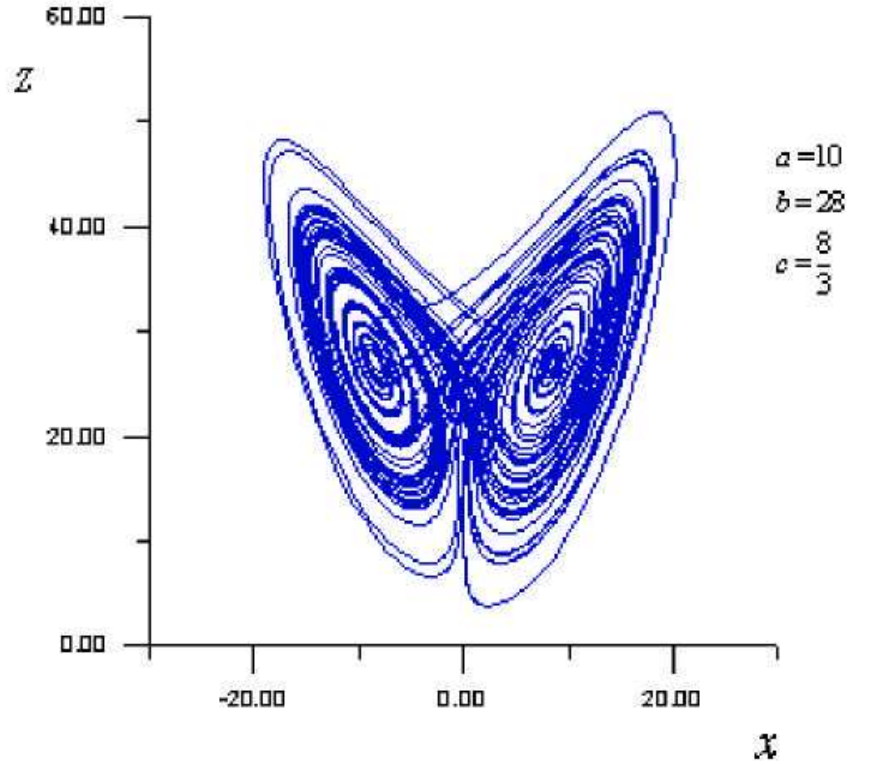

(a) attractors are shown. Lorenz system [Lorenz, 1963] has the following expression

$$
\begin{aligned}
& \dot{x}=a(y-x) \\
& \dot{y}=b x-y-x z \\
& \dot{z}=x y-c z
\end{aligned}
$$

and the Rossler one [Rossler, 1976] is described by

$$
\begin{aligned}
& \dot{x}=-(y+z) \\
& \dot{y}=x+a y \\
& \dot{z}=b+z(x-r) .
\end{aligned}
$$

The correlation studies for Lorenz and Rossler attractors are shown in Figs. 20 and 21, respectively.

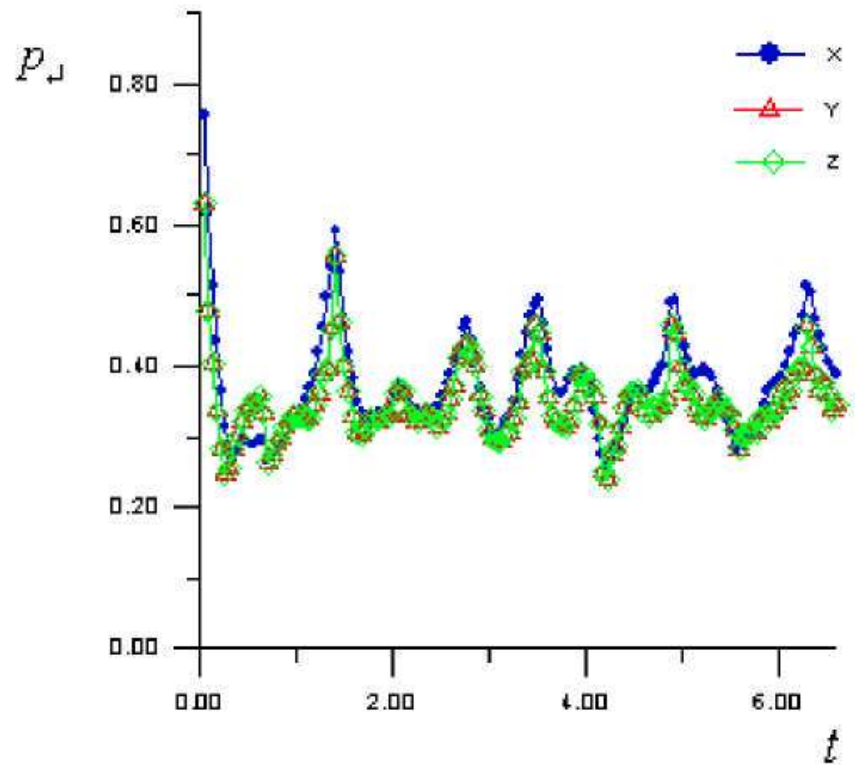

(b)

Fig. 20. Lorenz attractor (a). Correlation study (b). 


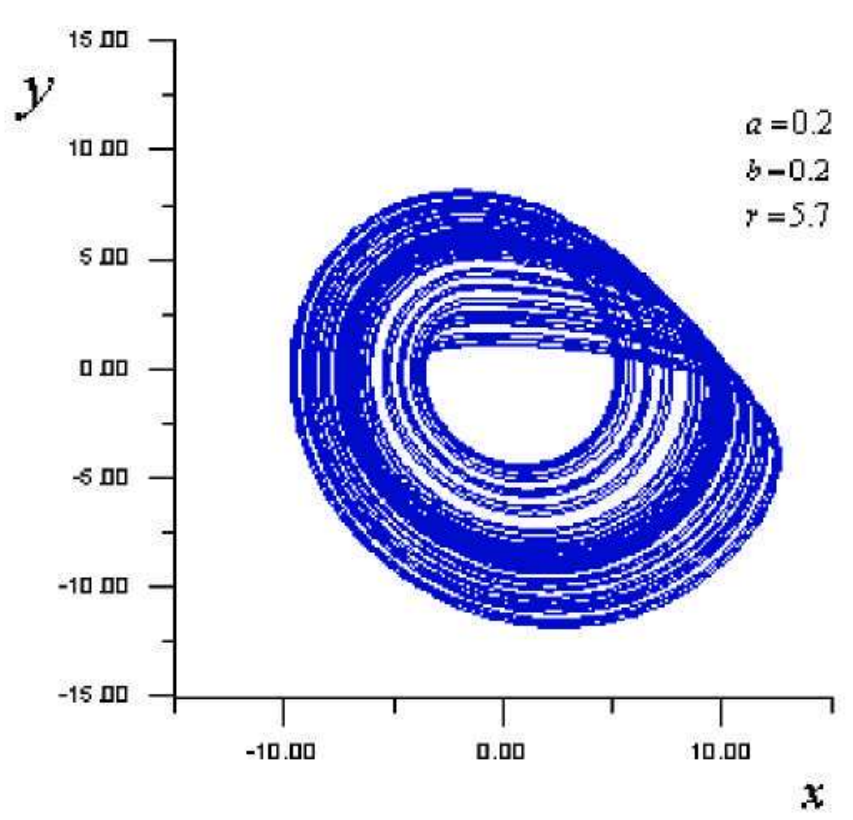

(a)

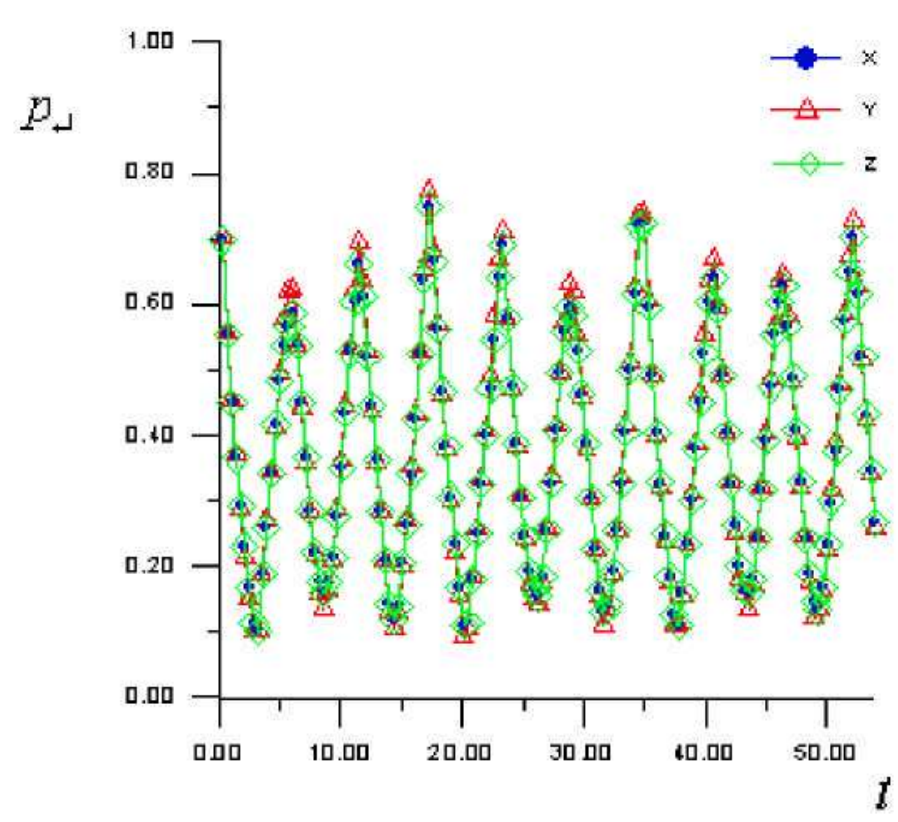

(b)

Fig. 21. Rossler attractor (a). Correlation study (b).

The parameter values for the systems (17) and (18) are specified in the figures. It is important to detail the process for calculating $p_{\perp}$ with continuous series. An original series $s^{*}(t)=$ $s^{*}(\triangle t), s^{*}(2 \triangle t), \ldots, s^{*}(N \triangle t)$, belonging to one of the variables of the system, is obtained by using any standard method, for instance Runge-Kutta, with certain integration step $\Delta t$. After that, a sampling period $\delta$ is selected to obtain the final series $s(t)=s(\delta \Delta t), s(2 \delta \Delta t), \ldots, s(N \delta \Delta t)$. If $s^{*}(t)$ is long enough to describe the general evolution of the variable in the attractor, $s(t)$ is, besides being shorter, also a rough description of the variable. In other words, if $s^{*}(t)$ has enough points to visit the vicinity of entire regions of the attractor, the sampled series $s(t)$ also describes the general evolution. Working with $s(t)$ instead of $s^{*}(t)$ has the advantage of obtaining the same results operating with a shorter series. Hence, $p_{\perp}$ can be calculated directly with $s^{*}(t)$ or $s(t)$, saving computation time.

As can be seen in Figs. 20 and 21 the same structures of $p_{\perp}$ are found for all the variables in Lorenz and Rossler attractors. The relationship among the variables of the system seems to cause coupling in their averaged "Perpendicular" product. Although a universal law of perfect coupling of $p_{\perp}$ for any generic system, supported by an analysis of a huge number of systems, is not provided in this work, strong evidence has been presented which point to the existence of such a law. In any case, a perfect coupling in the correlation study is not needed to carry out a characterization of the system with only one of its variables, since such characterization must be done with the dominant values of $\tau$. It means that only the dominant values of $\tau$ must be coupled for all the variables, which relaxes the necessary condition for dimension reduction.

\section{Application 2. Modeling of Experimental Time Series}

In order to prove that the method has a clear potential in the characterization of complex time series, a real application is presented in this section. Two time series are characterized and modeled by using the basic ideas proposed in this work. These series correspond to telephony traffic belonging to a Spanish telecom company. It is a national telephony network interconnected with other national networks in the most important provinces of the country. Likewise, it has an interconnection with an international carrier to route towards international destinations. Calls distribution scheme is as follows: calls originated from any customer with destination to a certain province are routed to the corresponding provincial "point of interconnection", from now on "poi". The call is delivered there to another operator, provided that the destination number does not belong to the own network. These provincial "pois" have two-way traffic, since incoming calls are 
already received from external networks. In the case of incoming or outgoing international calls the international "poi" is used. A "poi" consists of interconnection hardware, which is used to allocate calls and its occupancy can be measured in real time. This occupied capacity, due to incoming and outgoing traffic, is a function of time and it is usually measured in "Erlangs" [Erlang, 1909]. So, the amount of traffic routed in a unit of time through a single poi is a time series. The modeling of traffic patterns is very important as it can be used for network planning purposes. Two time series are studied, on the one hand provincial traffic through a single provincial poi, on the other hand international traffic through an international poi. Provincial and international traffic series have been selected to verify if the study can reveal different internal dynamics. The time unit is a complete day and the traffic one is the Erlang. The length of both series is 365 data corresponding to the traffic measured by the operator throughout a year. Figure 22 shows the series under study without any filtering process. Note that provincial traffic is quite regular with a light upward trend whereas international traffic is really irregular with also a late upward trend.

\subsection{Correlation study}

The graphs at the bottom in Fig. 18 show the correlation study for both series. Figure 18(a) corresponds to the provincial series and Fig. 18(b) to the international one. A clear pattern arises in both figures. There is a strong pseudo-periodicity for $T=7$, which is logical considering the weekly periodicity of the activities in the human society. Note that the multiples of $\tau=7$ are kept important without information loss. The result is specially interesting for the international traffic since its time series seems to be really disordered, however its correlation study reveals a clear underlying pseudo-periodicity.

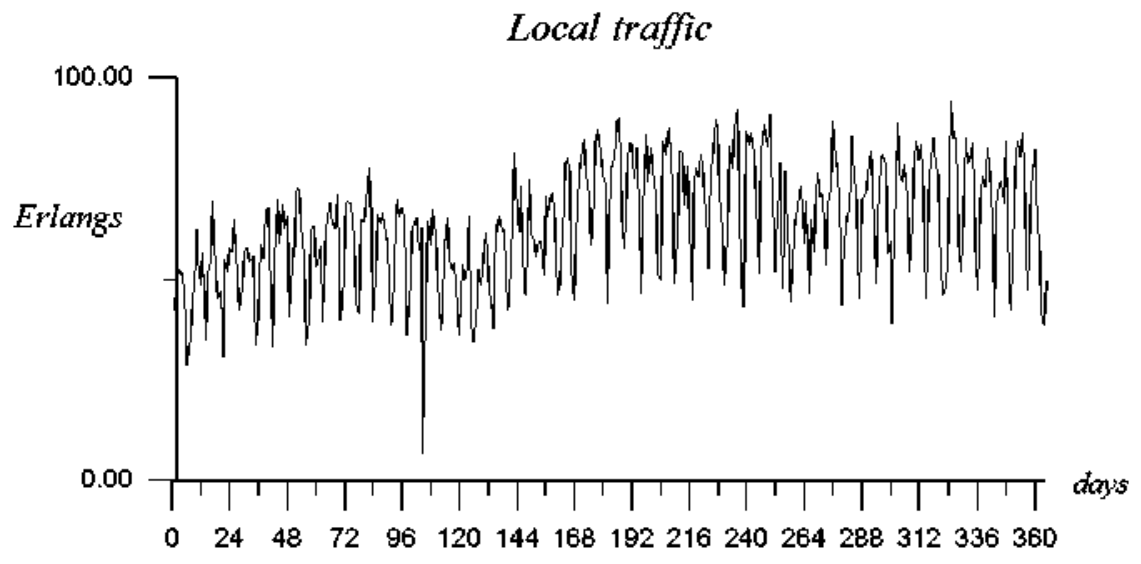

(a)

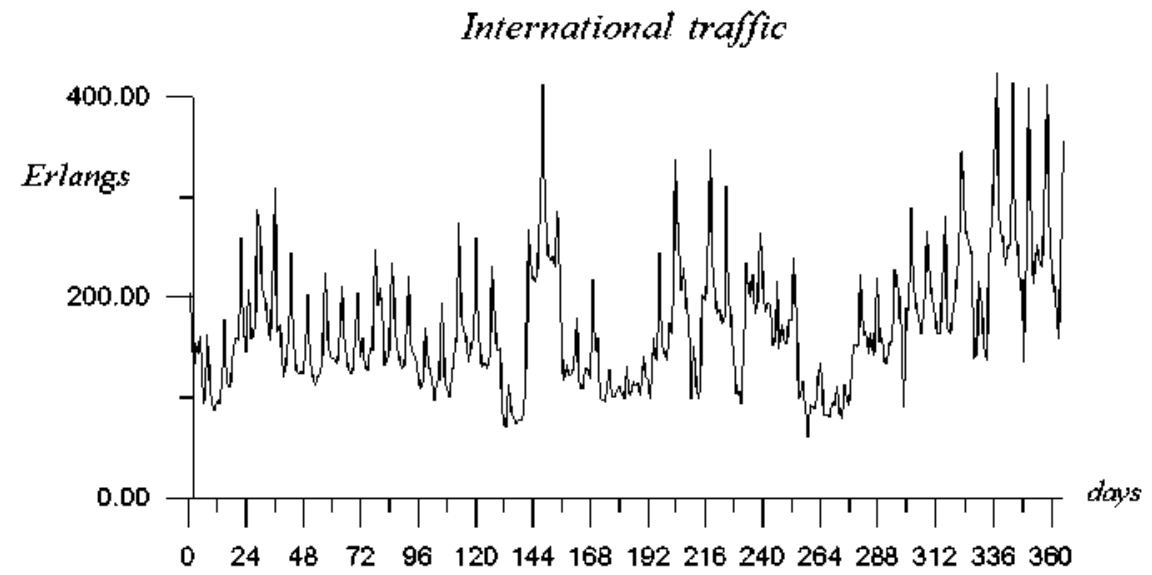

(b)

Fig. 22. Time series of (a) provincial traffic and (b) international traffic. 


\subsection{Modeling of the traffic series}

Once the self-correlation has been characterized, the purpose will be to use this information to obtain an equation which describes correctly the time series. As "Perpendicular" product measures periodicity and pseudo-periodicity, it is logical to use an equation in differences built with the predominant values of $\tau$. The next model is proposed:

Choose, according to the correlation study, five predominant values of $\tau,\left\{\tau_{j}\right\}$ with $j=1,2, \ldots, 5$ and consider the next equation as a model of the time series

$$
x_{k}=\phi_{m} x_{k-\tau_{m}} .
$$

Calculate $\phi_{j}^{k}=x_{k-\tau_{j}} / x_{k-2 \tau_{j}}$ for every $j$, given a certain $k$. Select the minimum value of $\{\mid 1-$ $\left.\phi_{1}^{k}|| 1-,\phi_{2}^{k}|, \ldots| 1-,\phi_{5}^{k} \mid\right\}$ and write its corresponding $\phi_{j}^{k}$ as $\phi_{m}^{k}$.

Hence, only one $\tau_{j}$ is used in (19) for every $k$. It means that the model considers the underlying dynamics as pseudo-periodic, hopping among five different pseudo-periods. This is only an example of model, although other choices may be defined by using the information derived from the correlation study of a time series. The arbitrary criterion for the selection of $\phi_{m}^{k}$ flows from the hypothesis of "equilibrium state". The system "decides" to keep as close to the equilibrium as possible for every $k$. Such equilibrium implies not to generate either upward or downward trends, or at least generate the slightest possible trend, which is reached with $\phi_{j}^{k}$ closest to 1 . Note that $\phi_{j}^{k}$ is the growth rate for the period $\left[k-2 \tau_{j}, k-\tau_{j}\right]$, and (19) supposes the same growth rate for the next period $\left[k-\tau_{j}, k\right]$.

Taking into account these considerations, Eq. (19) can be rewritten as

$$
x_{k}=\frac{x_{k-\tau_{m}}^{2}}{x_{k-2 \tau_{m}}}
$$

which is a nonlinear equation in differences. Suppose that the previous expression describes the underlying dynamics, then the prediction model is

$$
\hat{x}_{k}=\frac{x_{k-\tau_{m}}^{2}}{x_{k-2 \tau_{m}}}
$$

where $\hat{x}_{k}$ is the predicted value in $t=k$, and $x_{k-\tau_{m}}, x_{k-2 \tau_{m}}$ are values obtained from the real series. Note that the prediction horizon matches with the minimum value of $\left\{\tau_{j}\right\}$.

In order to reconstruct both, provincial and international traffic series, the first five multiples of 7 are chosen, $\tau=7, \tau=14, \ldots, \tau=35$, basing the selection on the previous correlation study with a strong weekly pseudo-periodicity. The reconstruction of the provincial traffic series is depicted in Fig. 23 according to (21). The model is shown to describe quite well the evolution of the telephony traffic with a small averaged normalized error $\left(E=\left\langle\left|x_{k}-\hat{x}_{k}\right| / x_{k}\right\rangle=0.149\right)$. The reconstruction of the international traffic series is shown in Fig. 24. Although the model adjusts less in this case, due to the higher complexity of the series, it can be seen in the green square that the model follows the same

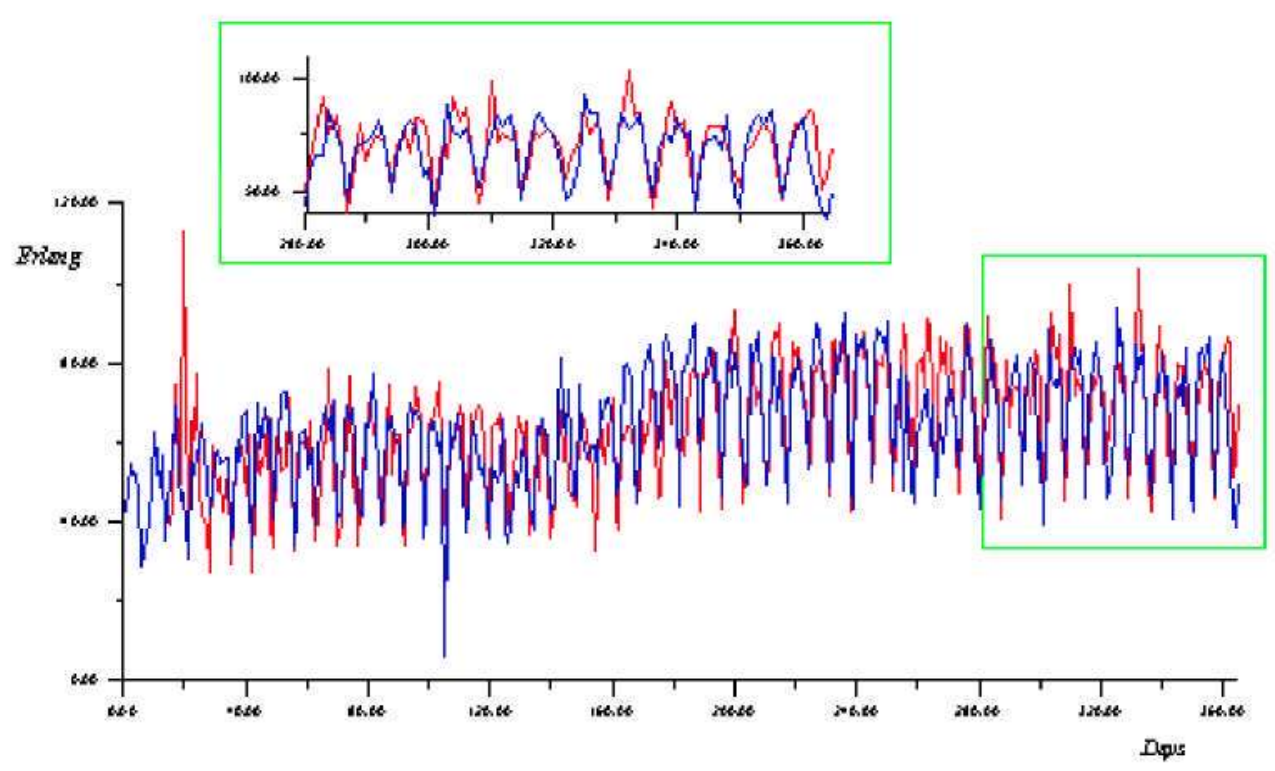

Fig. 23. Comparison of the time series of the provincial traffic (blue) with the proposed model (red). 

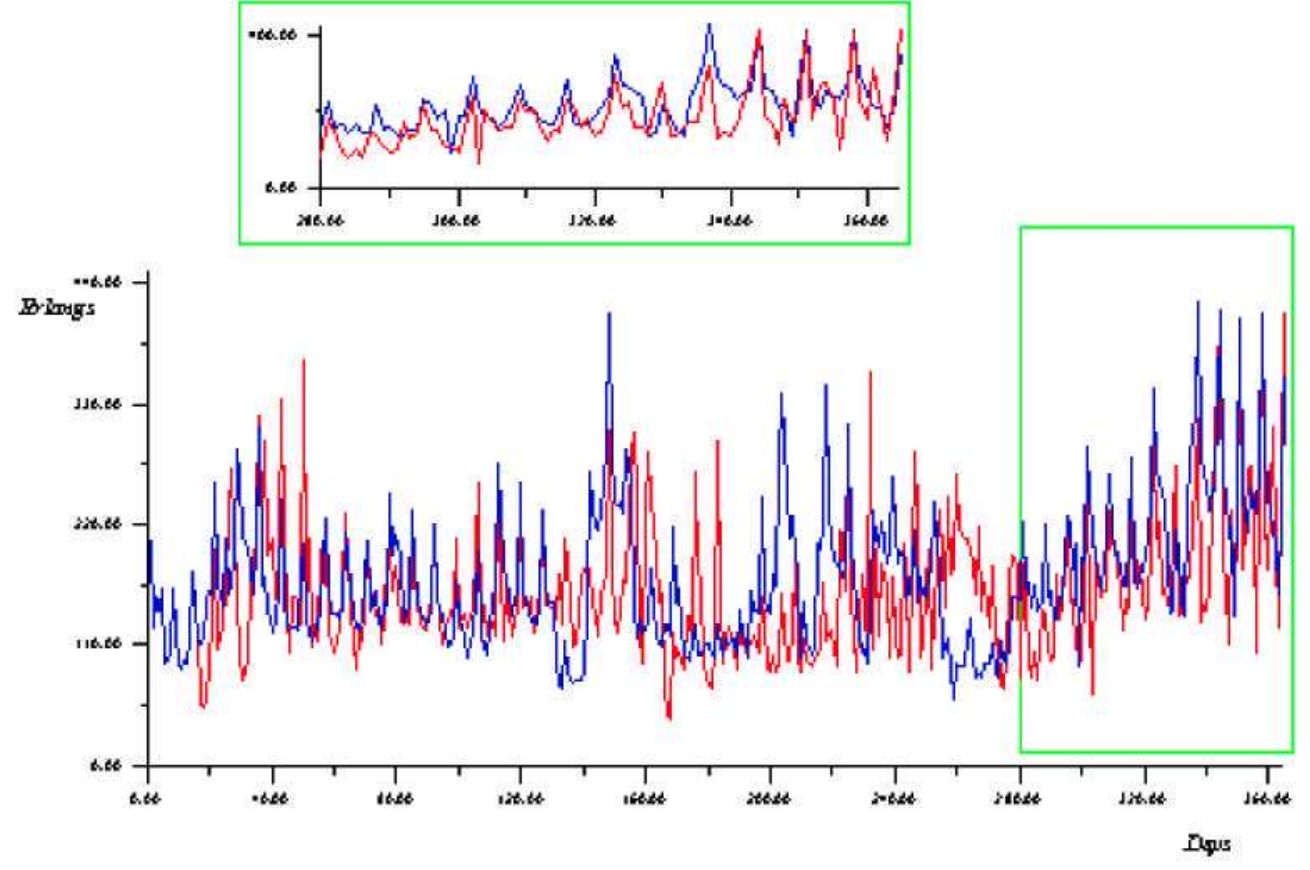

Fig. 24. Same as Fig. 23 for international traffic.

pseudo-periodic evolution as the real series and still provides an acceptable averaged normalized error $(E=0.337)$.

\section{Conclusions}

We have introduced two normalized indicators, the averaged "Perpendicular" and "Scalar" products, which combined together have shown to be a complete method to characterize nonlinear systems and a basis for modeling. These indicators are measures of "Self-correlation" and "Dynamical order" respectively. The first concept is a sort of correlation which detects how periodic or pseudo-periodic a series is whereas the second concept is a measure of how ordered the states move within a state space. The "Perpendicular" product provides a spectrum (correlation study), when it is calculated over a range of $\tau$, forming the real characterization of the system. In fact, the method could be supported only on the "Perpendicular" product but then a complete correlation study should be carried out for every value of the parameters in a system. "Scalar" product implies calculation only once, for $\tau=1$, so it is a fast indicator of order with minimum computation time. Hence, the combination of both measures is optimal from a computation point of view. Although another measure has been defined, the socalled "Escape velocity", in fact it is a derivation from the "Perpendicular" product, so the method is still based on two basic indicators.

An important aspect is the dimension reduction. If the $m$ variables of an $m$-dimensional system have coupled correlation studies, it is enough to carry out the characterization for only one of its variables. Even if this coupling is not perfect, but only the predominant $\tau$ are coupled, the statement is still valid: the study on just one variable is enough. Some results have been shown in this work which prove that several classical chaotic systems have a perfect coupling, which seems to point to a more general law.

The algorithm of "Equilibrium state" has been proposed to address the problem of modeling. Such algorithm uses the predominant $\tau_{j}$ from the "Correlation study" to build a model with equations in differences. The evolution of this model consists of transitions among pseudo-periodic states $\left(T \approx \tau_{j}\right)$ with the "Equilibrium hypothesis" as the internal controller of such transitions.

Two significant conclusions are derived from the introduced methodology. On the one hand, the "Scalar" product can be used as a supplement of the "Lyapunov exponent" to distinguish among different dynamical states when the "Lyapunov exponent" cannot detect subtle differences. Its sensitivity to slight dynamical changes can be used to provide further information, differentiating close 
dynamical states when the "Lyapunov exponent" reaches its maximum resolution. On the other hand, "Perpendicular" product plays an important role in the context of nonlinear correlation. When comparing "Perpendicular" product with the Auto Mutual Information (AMI), the former has been shown to be a refinement of the latter, after it has reached its first minimum.

\section{Acknowledgments}

We thank Dr. Manuel Maldonado for his useful advice and constant support. This work was supported by the Spanish Ministry of Science, under Projects No. MTM2009-14621 and Ingenio Mathematica (i-MATH) No. CSD2006-00032.

\section{References}

Amigo, J. M., Kocarev, L. \& Szczepanski, J. [2006] "Order patterns and chaos," Phys. Lett. A 355, 2731 .

Amigo, J. M., Elizalde, S. \& Kennel, M. B. [2008] "Forbidden patterns and shift systems," J. Comb. Theory A 115, 485-504.

Badii, R. \& Politi, A. [1986] "On the fractal dimension of filtered chaotic signals," Dimensions and Entropies in Chuotic Systems (Springer-Verlag), p. 67 .

Bennetin, G., Galgani, L. \& Streclyn, J. M. [1976] "Kolmogorov entropy and numerical experiments," Phys. Rev. A 14, 2338-2345.

Carpi, L., Saco, P. \& Rosso, O. A. [2010] "Missing ordinal patterns in correlated noises," Physica A 389, 20202029 .

Cellucci, C. J. \& Albano, A. M. [2005] "Statistical validation of mutual information calculations: Comparison of alternative numerical algorithms," Phys. Rev. E 71, 066208.

Eckmann, J. P., Oliffison, K. S., Ruelle, D. \& Ciliberto, S. [1986] "Lyapunov exponents from a time series," Phys. Rev. A 34, 4971-4979.

Erlang, A. [1909] "The theory of probabilities and telephone conversations," Nyt Tidsskrift for Matematik $B$ $20,33-39$.

Fraser, A. \& Swinney, H. [1986] "Independent coordinates for strange attractors from mutual information," Phys. Rev. A 33, 1134-1140.

Fridrich, J. [1998] "Symmetric ciphers based on twodimensional chaotic maps," Int. I. Bifurcation and Chaos 6, 1259-1284.

Gottwald, G. A. \& Melbourne, I. [2009] "On the implementation of the 0-1 test for chaos," SIAM J. Appl. Dyn. Syst. 8, 129-145.
Grassberger, P. \& Procaccia, I. [1983] "Estimation of the Kolmogorov entropy from a chaotic signal," Phys. Rev. A 28, 2591-2593.

Grassberger, P. [1987] "Estimating the fractal dimensions and entropies of strange attractors," Chaos (Manchester University Press), p. 291.

Guegan, D. [2009] "Chaos in economics and finance," Ann. Rev. Contr. 33, 89-93.

Halbiniak, Z. \&. Jozwiak, I. J. [2007] "Deterministic chaos in the processor load," Chaos Solit. Fract. 31, $409-416$.

Hausdorff, F. P. [1919] "Dimension und ausseres mass," Math. Annalen 79, 157-179.

Hénon, M. [1976] "A two-dimensional mapping with a strange attractor," Commun. Math. Phys. 50, $69-77$.

Hénon, M. [1982] "On the numerical computation of Poincaré maps," Physica D 5, 412-414.

Huerta, R., Santa Cruz, C., Dorronsoro, J. R. \& López, $\mathrm{V}$. [1994] "State space reconstruction using averaged scalar products of the dynamical system flow vectors," Phys. Rev. E 49, 1962-1967.

Khan, S. et al. [2007] "Relative performance of mutual information estimation methods for quantifying the dependence among short and noisy data," Phys. Rev. E 76, 026209.

Karakasidis, T. E. \& Charakopoulos, A. [2009] "Detection of low-dimensional chaos in wind time series," Chaos Solit. Fract. 41, 1723-1732.

Kolmogorov, A. N. [1959] "Entropy per unit time as a metric invariant of automorphisms," Doll Akad. Nauk SSSR 124, p. 754

Laurent, M., Deschatrette, J. \& Wolfrom, C. M. [2010] "Unmasking chaotic attributes in time series of living cell populations," PLOS ONE 5, 9346-9358.

Li, C. \& Chen, G. [2004] "Estimating the Lyapunov exponents of discrete systems," Chaos 14, 343-346.

López, R. \& Pérez, C. [1991] "Dynamics of maps with a global multiplicative coupling," Chaos Solit. Fract. 6, 511-528.

López, R. \& Fournier, D. [2003] "Complex patterns on the plane: Different types of basin fractalization in a two-dimensional mapping," Int. J. Bifurcation and Chaos 13, 287-310.

Lorenz, E. N. [1963] "Deterministic nonperiodic flow," J. Atmos. Sci. 20, 130-141.

Mandelbrot, B. B. [1983] The Fractal Geometry of Nature (W. H. Freeman \& Co.).

May, R. M. [1976] "Simple mathematical models with very complicated dynamics," Nature 261,459-467.

Okushima, T. [2003] "New method for computing finitetime Lyapunov exponents," Phys. Rev. Lett. 91, 254101 .

Poincaré, H. [1892] Les Méthodes Nowolles de la Mécanique Céleste (Gauthier-Villars et fils). 
Rogers, C. A. [1970] Housdorff Measures (Cambridge University Press).

Rossler, O. E. [1976] "An equation for continuous chaos," Phys. Lett. A 57, 397-398.

Rosso, O. A., Larrondo, H. A., Martin, M. T., Plastino, A. \& Fuentes, M. A. [2007] "Distinguishing noise from chaos," Phys. Rev. Lett. 99, 154102-154105.

Sano, M. \& Sawada, Y. [1985] "Measurement of the Lyapunov spectrum from a chaotic time series," Phys. Rev. Lett. 55, 1082-1085.

Schleicher, D. [2005] "Hausdorff dimension, its properties, and its surprises," Am. Math. Mon. 114, 509528 .

Shannon, E. \& Weaver, W. [1949] The Mathematical Theory of the Communication (University of Illinois Press).

Shao, C., Fan, J. \& Chen, X. [2007] "Extracting qualitative states from nonlinear time series using integration of fuzzy $c$-means and hierarchical clustering," 4 th Int. Conf. Fuzzy Systems and Knowledge Discovery 2 , pp. 235-239.

Stoop, R. \& Meier, P. F. [1988] "Evaluation of Lyapunov exponents and scaling functions from time series," Opt. Soc. Am. B 5, 1037-1045.
Wolf, A., Swift, J. B., Swinney, H. L. \& Vastano, J. A. [1985] "Determining Lyapunov exponents from a time series," Physica D 16, 285-317.

Xie, H. B., He, W. X. \& Liu, H. [2008] "Measuring time series regularity using nonlinear similarity-based sample entropy," Phys. Lett. A 372, 7140-7146.

Xie, H. B., Guo, J. Y. \& Zheng, Y. P. [2010] "Using the modified sample entropy to detect determinism," Phys. Lett. A 374, 3926-3931.

$\mathrm{Xu}, \mathrm{X}$, Zhang, J. \& Small, M. [2008] "Superfamily phenomena and motifs of networks induced from time series," Proc. Natl. Acad. Sci. USA 105, 19601-19605.

Zanin, M. [2008] "Forbidden patterns in financial time series," Chaos 18, 013119-013122.

Zhang, J. \& Small, M. [2006] "Complex network from pseudoperiodic time series: Topology versus dynamics," Phys. Rev. Lett. 96, 238701-238704.

Zhang, J., Sun, J., Luo, X., Zhang, K., Nakamura, T. \& Small, M. [2008] "Characterizing pseudo-periodic time series through the complex network approach," Physica D 237, 2856-2865. 